\title{
G

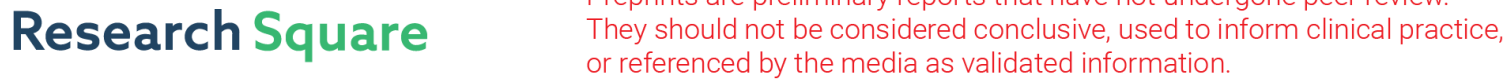 \\ Gadollium Nanoparticles Delivery for Low Intensity Focused Ultrasound Diagnosis Ablation of Thyroid Cancer Therapy
}

\author{
Ming Qi \\ JinZhou Medical College: Jinzhou Medical University \\ Yufeng Zhu \\ JinZhou Medical College: Jinzhou Medical University \\ Wenjuan Wang \\ JinZhou Medical College: Jinzhou Medical University \\ Shufeng Gao \\ JinZhou Medical College: Jinzhou Medical University \\ Sihui Nie \\ JinZhou Medical College: Jinzhou Medical University \\ Ke Wang \\ JinZhou Medical College: Jinzhou Medical University \\ Lingling Guo ( $\nabla$ guolingling99@yahoo.com ) \\ Jinzhou Medical University
}

\section{Nano Express}

Keywords: Chemotherapeutic efficacy, nanotheranostics systems, low intensity focused ultrasound diagnosis imaging (LIFUS)

Posted Date: December 1st, 2020

DOl: https://doi.org/10.21203/rs.3.rs-114205/v1

License: (c) (1) This work is licensed under a Creative Commons Attribution 4.0 International License. Read Full License 


\section{Abstract}

Chemotherapeutic efficacy can be significantly developed nanotheranostics systems of drug delivery in tumor cells. In this work, we have demonstrated that the self-assembled by C225 conjugates Gd-PFH-NPs (C-Gd-PFH-NPs) for low intensity focused ultrasound diagnosis ablation of thyroid cancer treatment. CGd-PFH-NPs have shown excellent stability in PBS. Transmission electron microscopy (TEM) images also exposed the effective construction of C-Gd-PFH-NPs with commonly spherical sized assemblies. The incubation of the $\mathrm{C} 625$ thyroid carcinoma with C-Gd-PFH-NPs triggers apoptosis, which was confirmed by the flowcytometry analysis. The C-Gd-PFH-NPs, with remarkably displays the potent antitumor efficacy in a human $\mathrm{C} 625$ thyroid carcinoma xenografts. A histopathological result reveals that precisely achieved to additional confirm these outcomes. Further, we successfully examined the efficiency of C-Gd-PFH-NPs when used the thyroid carcinoma low intensity focused ultrasound diagnosis imaging (LIFUS) in vivo. These findings clearable for LIFUS agents with high performing image and different therapeutic purpose will have extensive possible for the future biomedical purposes.

\section{Introduction}

In a recent time, triggerable drug-charged nanocarriers coupled with multiple inner or external stimuli such as $\mathrm{pH}$, temperature, ultrasound, laser, and microwave radiation have been extensively explored for personalized treatment to enable controlled release and have excellent possible to deliver an enhanced anticancer treatment impact also decreased systemic toxicity [1-4]. Low-intensity focused ultrasounds (LIFUS) have been exhaustively researched for tumour treatment and ultrasounds imaging analysis as one of the probable exterior activates which is non-invasive and displays significant tissue-penetrating capacity. In particular, it can significantly increase the efficacy of chemotherapy, avoiding harm to the nearby cells and reducing adversarial side effects [5-8]. Though, the discharge of LIFUS-triggered drugs from nanocarriers and further tumour therapy is still unsatisfactory, largely attributable to the comparatively less accumulation efficacy of nanoparticles-charged nano transporters at tumour places. There are numerous nanotransporters extensively examined on this basis to enhance the aggregation of large number of tumors without causing any side effects [9-12]. Anaplastic thyroid carcinoma (ATC) is one of the most malignant carcinomas, which is also comparatively rare, characterized by fast proliferation, neck invasion, and remote metastasis. ATC's severe prognosis is due to the tumors' fast progression before diagnosis [13-15]. Current treatment is based on different types of combinations in chemotherapy and exterior ray radiation has unsuccessful to enhance existence, resulting in an average existence rate of 4 to 6 months and less than $20 \%$ existence level in 12 months. Here are therefore convincing explanations for developing a new theranostics approach for initial finding and efficient ATC treatment [16-19].

Several reports have shown that overexpression of the epidermal growth factor receptor (EGFR) is strongly associated with tumour progression, migration, and invasion. EGFR is common in ATC. Antibodies or small molecules based on EGFR immunotherapy can significantly increase the therapeutic effect against ATC. A human murine chimeric EGFR-targeted monoclonal antibody called Cetuximab 
have higher empathy to human EGFR's extracellular domain and inhibits the signals of its epidermal growth factor in cells by delaying usual receptor function [20-22]. Food and Drug Administration approved preclinical and preclinical treatments using Cetuximab for the treatment of EGFR-expressing cancer tumors' neck and head carcinoma and colorectal carcinoma. This C225 might be a suitable objective for the nanocarriers' structure to improve the therapeutic outcome of ATCs. Remarkably, some researchers have revealed that for a wide spectrum of cancers, the blend of C225 with CPT-11 equivalents such as Gd-PFH-NPs has significant synergetic antitumor effects [23-25]. Hence, Gd-PFH-NPs in combination with $\mathrm{C} 225$ could enhance the ATC diagnostics. But, owing to less vascular dispersal of C225 and hydrophobicity of the Gd-PFA-NPs, the NPs penetrability in the growth and the NPs quantity in the tumor area were inherently imperfect, shows greatly debilitated their anticancer efficacy [26,27]. Opportunely, the problems can be enhanced through incorporating Gd-PFH-NPs and C225 into a one nanotransporters to attain C225 and Gd-PFH-NPs combination chemotherapy while simultaneously providing targeting capability for nanocarriers $[24,25,28,29]$.

Furthermore, for early diagnosis and tumor progression monitoring medical imaging is essential. Numerous researchers proposed that LIFUS have the probable to achieve concurrent US and medication transfer, meeting the present need for initial treatment and ATC therapy [30-32]. Due to variability and huge dimensions of microbubbles to realize the tumor theranostic strategy conservative US agents, like as microbubbles, demonstration outstanding US agents for imaging capability but these not appropriate for drug delivery purposes. In order to avoid this problem, intensively studied phase-changing NPs that could be activated via LIFUS [33-35]. The phase-changing NPs providing important benefits in tumor theranostics for the supply of tumor ultrasound and ultrasound-triggered drug. This new strategy offers the possible to develop malignancy treatment and addresses the present theranostic needs in contradiction of ATC significantly.

The objective of this work was to constructed the modification of C225 nanocarrier to exactly prevent ATC that might accrue in cancer cells, in addition to the EPR effect, through the great tumor homing belongings of C225. The Gd-PFH-NPs payload could be released and LIFUS-triggered synergistic chemotherapy with C225 may perhaps suggestively make best use of therapeutic efficacy, improve USI and diminish the side effects of chemotherapy. As shown in Fig. 1. Due to its tremendous biodegradability and biocompatibility, we used a PHF (Perfluorohexane) core as the shell structure of the nanocarrier. We then synthesized phase-changing NPs with Perfluorohexane liquid (PHF, $29^{\circ} \mathrm{C}$ boiling point). Meanwhile, Gd-PFH-NPs were burdened into the nanoparticles at the similar period of time as C225 was conjugated on surface of manganese nanoparticles afford (C-Gd-PFH-NPs) C225-conjugated Gd-PFA-NPs-charged phase transformation. To our knowledge, this is the first work of a LIFUS-mediated C225 modified nanosyste that assimilates tumor targeted both US imagery and US activated drug conveyance to ATC.

\section{Experimental Section}

\subsection{Characterization of C-Gd-PFH-NPs}


ptical microscopy ( CKX41; Olympus, Tokyo, Japan) and confocal laser scanning microscopy (CLSM) ( Nikon A1, Tokyo, Japan ) have observed the morphology and particle distribution of Gd-PFH-NPs and CGd-PFH-NPs. A dynamic light scattering analyzer ( DLS) (Malvern Instruments, Malvern, UK) was used to determine the mean particle size, polydispersity index (PDI) NPs. Using transmission electron microscopy (TEM) ( H-7500; Hitachi, Tokyo, Japan) the morphological characterization of NPs were carried out. The mean particle size of the nanoparticles was determined by DLS tested within 7 days in order to better illuminate the stability of the Gd-PFH-NPs and C-Gd-PFH-NPs.

\subsection{Synthesis of C-Gd-PFH-NPs}

Gd and PFH nanoparticles (Gd-PFH-NPs) were fabricated by a film hydration method coupled with a double emulsion method [36-39]. $100 \mathrm{~mL}$ of $\mathrm{Gd}$ solution $(10 \mathrm{mg} / \mathrm{mL})$ were added to the $\mathrm{CHCl}_{3}$ solution. The mixture was transferred into the rotary evaporator (Yarong Inc., Shanghai, China) and rotated at $55 \mathrm{rpm}$ and $45^{\circ} \mathrm{C}$ to clear the $\mathrm{CHCl}_{3}$. The thin lipid film formed was rehydrated with $5 \mathrm{~mL}$ PBS to generate a brown suspension. The suspension was then dispersed with a high-speed homogenizer (FJ300-SH, Shanghai, China) for $5 \mathrm{~m}$ after drop-by-drop addition of $500 \mathrm{~mL}$ PFH. The secondary emulsion was performed by means of an ultrasonic oscillator (SONICS \& MATERIALS Inc., USA) for $5 \mathrm{~m}$ in an icecold environment $\left(0^{\circ} \mathrm{C}\right)$. Finally, the mixture of nanoemulsions was harvested and centrifuged (Eppendorf, Germany) at $4500 \mathrm{rpm}$ for $5 \mathrm{~m}$ and washed with deionized water for three times to sweep away dissociative $\mathrm{Gd}$ and PFH. The final emulsion was collected and stored at $4{ }^{\circ} \mathrm{C}$ for further use.

Fluorescent nanoemulsions were obtained according to the above procedure except that the Dil was blended in the lipids solution.

\subsection{C225 Conjugation}

Conjugation of $\mathrm{C} 225$ to the Gd-PFH-NPs loaded nanoparticles was performed using carbodiimide chemistry. Briefly, the prepared HPNs were dissolved in $5 \mathrm{~mL}$ of MES buffer solution (0.1 M, pH 5.5) together with a mixture of $3 \mathrm{mg}$ of EDC and $10 \mathrm{mg}$ of NHS, and then incubated vigorously for a period of $1 \mathrm{~h}$ on a gentle shaker. The resulting solution was centrifuged and washed three times with PBS to remove unreacted EDC and NHS. Then, the sediment was redissolved in $5 \mathrm{~mL}$ of MES buffer solution (0.1 M, pH 8.0). Next, excess $\mathrm{C} 225$ was dropped into the above solution and stirred on a gentle shaker for another $2 \mathrm{~h}$. After the reaction was completed, Gd-PFH-NPs were obtained by centrifugation, washed thrice with PBS again to remove unconjugated $\mathrm{C} 225$ and preserved at $4{ }^{\circ} \mathrm{C}$ before use. All the aforementioned procedures were carried out in an ice bath. PNs with C225 conjugation (C-Gd-PFH-NPs) were also prepared using the same procedures.

\subsection{Cell Culture and Nude Mice}

The Cell Bank of the Chinese Academy of Sciences (Shanghai, China) acquired a human anaplastic thyroid carcinoma line (C643). The cells were grown in medium RPMI-1640 containing 10\% FBS and $1 \%$ penicillin-streptomycin at $37^{\circ} \mathrm{C}$ in humidified air with $5 \%$ CO2. BALB/C Female both mice and nude mice (balancing about $19 \mathrm{~g}, 25$ days) were bought then raised. All animals on our studies were collected from 
the Ultrasound Department, The First Affiliated Hospital of Jinzhou Medical University Laboratory Animal Center and retained in accordance with rules authorized by the First Affiliated Hospital of Jinzhou Medical University Animal Ethics Committee (Harbin, China). Furthermore, all animal experimental activities were strictly in line with the policy of the Harbin Medical University's Institutional Animal Care and Use Committee (IACUC), and this study was endorsed by the IACUC.

In order to start an ATC model in nude mice, C643 cells were collected, splashed thrice with the FBS free medium of RPMI-1640, and subcutaneously inoculated into each mouse's left flank ( $3 \times 107$ C643 cells in $150 \mu \mathrm{L}$ FBS free medium of RPMI-1640 each mice). A Vernier caliper was used to measure the length and width of the tumour and the tumour quantity was considered by the calculation: volume-(length as width $\times 2) / 2$.

\subsection{In Vitro Intracellular Uptake C-Gd-PFH-NPs}

In cultivation dishes, seeded the C643 cells for CLSM at a mass of $1 \times 10^{6}$ cell $\mathrm{mL} /$ dish, grown at $37^{\circ} \mathrm{C}$ in moistened air comprising $5 \% \mathrm{CO}_{2}$. The cells were spilt into four groups after $24 \mathrm{~h}$ of culture: C-Gd-PFHNPs were handled respectively with $10 \mathrm{~min}$ and $15 \mathrm{~min}$ Dil- labeled C-Gd-PFH-NPs ( $1 \mathrm{mg} / \mathrm{mL})$, and after blocking the cells were washed three times with PBS. Then, Dil- labeled C-Gd-PFH-NPs ( $1 \mathrm{mg} / \mathrm{mL})$ incubated the cells. The cells were washed with PBS three times after $2 \mathrm{~h}$ incubation with nanoparticles, fixed with 4 percent paraformaldehyde $(200 \mu \mathrm{L})$ for 15 minutes, and then gestated by DAPI $(10 \mu \mathrm{g} / \mathrm{mL}$, $200 \mu \mathrm{L})$ for $20 \mathrm{~min}$. Lastly, CLSM pictured the dishes.

\subsection{In Vitro Cytotoxicity Assay}

The CCK-8 assay assessed the cell viability [40-42]. C643 cells were seeded into 96-well plates $(1 \times 103$ cells per well, $100 \mu \mathrm{L}$ ). After 24- hours' incubation to assess the cell viability Gd-PFH-NPs and C-Gd-PFHNPs treated at levels of $10,5,2.5,1.25,0.625$ and $0.312 \mu \mathrm{M}$ for 24 hours. Gd-PFH-NPs and C-Gd-PFH-NPs cells were incubated for 24 hours. The positive control used as the untreated $\mathrm{C} 643$ cells. The in vitro cytoxicicty assay performed and the calculated made by the company manufactures guidelines.

\subsection{Apoptotic Staining}

The morphological changes of the $\mathrm{C} 643$ cells were examined by biochemical staining, including acridine orange-ethidium bromide (AO-EB) and Hoechst 33344 staining [43,44]. After incubating for $24 \mathrm{~h}$, the cells were seeded at a concentration of $1 \times 104$ onto 48 well plates. The cells were treated with Gd-PFH-NPs and C-Gd-PFH-NPs at $2.5 \mu \mathrm{M}$ concentration for $24 \mathrm{~h}$. On the following day, the staining solution was added. After incubating the plates with the staining solution, the plates were washed with PBS three times. Images were obtained using a fluorescence microscope (Accu Scope EXI-310) at a magnification of $20 x$.

\subsection{Flow Cytometry/Annexin V-PI Staining}

The flow cytometry examination was examined by using the Apoptosis Detection Kit of fluoresceinisothiocyanate (FITC) (Cell Signalling, China) utilized to confirm the apoptotic ratio of C643 
cells. The cells were treated with Gd-PFH-NPs and C-Gd-PFH-NPs at $2.5 \mu \mathrm{M}$ concentrations for $24 \mathrm{~h}$. The cells were washed thrice by using trypsin, and suspended in $1 \times$ binding buffer $(500 \mu \mathrm{L})$ with FITC Annexin $\mathrm{V}(5 \mu \mathrm{L})$ and of $\mathrm{PI}(10 \mu \mathrm{L})$. After 20 min incubation, the samples were analysed by flow cytometry. The obtained results were investigated with the BD FACS CantoTM II flow cytometer.

\subsection{Evaluation of the In Vivo Drug Toxicity}

The in vivo drug toxicity was investigated in ICR mice (4-5 weeks old). Healthy ICR mice were randomly divided into 5 groups ( $n=10$ mice per group). Drugs were injected through the tail vein on days 0,3 , and 6. Mice were injected with Gd-PFH-NPs (2.5, and $5 \mathrm{mg} / \mathrm{kg}$, Gd equivalent dose), C-Gd-PFH-NPs (2.5, and $5 \mathrm{mg} / \mathrm{kg}$ ). Saline were injected as a control. The body weights of the mice were recorded every three days.

\subsection{Histologic Analysis}

For histological analysis, the organs from the sacrificed mice were excised at the end of the treatments with various drugs. After being fixed in $4 \%$ formaldehyde and embedded in paraffin, the tumor tissues and organs were further sectioned into $5 \mu \mathrm{m}$ slices for hematoxylin and eosin (H\&E, Sigma) staining. The H\&E-stained tissues were imaged by fluorescence microscopy (Olympus, IX71).

\subsection{In Vivo Antitumor Activity}

BALB/C nude mice (4-5 weeks old) were used for the evaluation of the antitumor activities of the nanotherapies. The human prostate cancer cell line $\mathrm{C} 643$ was grown to $80 \%$ confluence in $90 \mathrm{~mm}$ tissue culture dishes. After cell harvesting, the cells were resuspended in PBS at $4{ }^{\circ} \mathrm{C}$ to reach a final concentration of $2.5 \times 10^{7}$ cells $/ \mathrm{mL}$. The right flanks of the BALB/c nude mice were subcutaneously injected with $200 \mu \mathrm{L}$ of a cell suspension containing $5 \times 10^{6}$ cells. At 14 days after implantation, the tumors reached approximately $60 \mathrm{~mm}^{3}$ in volume, and then the animals were randomly divided into five groups ( $\mathrm{n}=7$ mice per group). Mice bearing $\mathrm{C} 643$ tumor xenografts were injected intravenously with samples solutions (Gd-PFH-NPs at $5 \mathrm{mg} / \mathrm{kg}, \mathrm{C}-\mathrm{Gd}-\mathrm{PFH}-\mathrm{NPs}$ at $5 \mathrm{mg} / \mathrm{kg}$ ) three times on days 0,3 , and 6 . Saline were also injected as a control. Tumor volumes and body weights were monitored and recorded for 33 days. The lengths $(L)$ and widths $(W)$ of the tumors were measured with calipers, and the tumor volume was calculated by the following formula: $V=(L \times W 2) / 2$, where $W$ is shorter than $L$. Mice were sacrificed by $\mathrm{CO}_{2}$ inhalation at the endpoint of the study [45-47].

\subsection{Data Analysis}

The data analysis of different groups was conducted with one-way ANOVA in GraphPad Prism 5 software. The significant level were considered at $P<0.05$ and greatly significant at $P<0.001$. All data are presented as mean $\pm S D$. (Unless otherwise stated, $n=3$ ).

\section{Results And Discussion}

\subsection{Description of C-Gd-PFH-NPs}


Having these both compounds in hand, we have examined the TEM analysis of Gd-PFH-NPs (Fig. 1A) and C-Gd-PFH-NPs (Fig. 1B). we next tested whether they are able to recapitulate self-assembly behavior in aqueous solutions. For this purpose, we dissolved the C-Gd-PFH-NPs prodrugs in DMSO $(10 \mathrm{mg} / \mathrm{mL})$ and then rapidly injected them into deionized (DI) water under ultrasonication. This procedure allows us to validate the solution was found to be transparent and slightly bluish. Observation by electron microscopy revealed that the drug molecules self-assembled to form a spherical nanoparticle structure. DLS showed a single peak distribution of the nanoparticles. The average hydrodynamic diameter (intensity) of the $\mathrm{Gd}$ PFH-NPs was about $\sim 77.1 \mathrm{~nm}$, and the C-Gd-PFH-NPs was about $\sim 100.0 \mathrm{~nm}$ (Fig. 1B and D). However, there is a certain adhesion between the nanoparticles formed by the self-assembly of simple small molecule drugs. Therefore, we have miscible with many hydrophobic drugs by combining the prodrug with the appropriate amount of $\mathrm{C} 225$ molecules. These nano-assemblies are formed and have been widely used for in vivo drug delivery, aiming to solve the problem of adhesion and to optimize cancerspecific drug delivery. Then, we measured the stability of C-Gd-PFH-NPs with PBS which shows significantly stable size in various parameters (Fig. 1E and F). Taken together, although C-Gd-PFH-NPs can self-assemble to form nanoparticles, they may not be stable enough. Therefore, C225 nanoparticles loaded with Gd-PFH were investigated further to evaluate anticancer efficacy in vitro.

\subsection{In Vitro Intracellular Uptake}

As illustrated in Fig. 3, the much tougher red fluorescence derived from Dil-labeled C-Gd-PFH-NPs was additional obviously concentrated in the C-Gd-PFH-NPs group around the cytomembrane of C643 cells compared to the non- target and antagonistic groups. Furthermore, bigger quantities of red fluorescence were noted after exposure to C-Gd-PFH-NPs group. These findings stated that through the elevated tumour -homing characteristics of C225, C-Gd-PFH-NPs could fix tightly to C643 cells, and considerably encouraged intracellular uptake by the $\mathrm{C} 643$ cells. In the resentment group, C-Gd-PFH-NPs lost the capacity to objective the $\mathrm{C} 643$ cells because the congested by surplus free $\mathrm{C} 225$, leading in small levels of C-Gd-PFH-NPs around the cells and demonstrating that C-Gd-PFH-NPs desired targeting effectiveness was the outcome of the EGFR- mediated directing capacity.

\subsection{In Vitro Cytotoxicity Assay}

The CCK-8 assay assessed the cell viability of different NP formulations at distinct levels, showing a dose dependent model. The cell viability of nanoparticles in the analyzed dose range was noted at more than $80 \%$, level at $10 \mathrm{mg} / \mathrm{mL}$. The comparatively small insignificant viability proposed that the elevated biocompatibility of phase-changing nanoparticles was appropriate aimed at in vivo application. Reasonably, Gd-PFH-NPs and C-Gd-PFH-NPs cell viabilities decreased considerably as levels of C-Gd-PFHNPs also increased. In particular, the cell viability of the cells treated with C-Gd-PFH-NPs was the low at the same concentration, implying that the mixture of C-Gd-PFH-NPs could boost cytotoxicity synergistically. The cell viability of C-Gd-PFH-NPs. The remarkably improved cytotoxicity of C-Gd-PFHNPs may lead from the increased cell membrane permeability caused by the cavitation effect and the 
improved C-Gd-PFH-NPs at the objective place, which significantly increased the inhibitory impression of C-Gd-PFH-NPs on cell development.

\subsection{Morphological Changes in C643 Cancer Cells}

Dual staining AO-EB is a qualitative technique used to identify live, early, late apoptotic, and necrotic cancer cells using fluorescent images to observe morphological changes in the nucleus of cells. AO permeates the intacts membranes of usual and early apoptotic cell and binds to DNA, which fluoresces uniform green in normal cells and as patches in early apoptotic cells due to chromatin condensations. In difference, EB is only penetrable in the incapacitated membrane of late apoptotics and necrotics cell, where it fluoresces as bright orange patch through its bindings to DNA fragment or apoptotic bodies in late apoptotic cells, and as a unchanging orange fluorescence in the necrotic cell, due to have the nuclear changes in the morphology of viable cell. AO-EB-stained C643 cells were incubated with Gd-PFH-NPs and C-Gd-PFH-NPs for $24 \mathrm{~h}$. As presented in Fig. 4, the presence of orange with reddish fluorescence with chromatin fragmentation after treatment of $\mathrm{C} 643$ cells treated with Gd-PFH-NPs suggested that the C-GdPFH-NPs largely induced apoptosis in $\mathrm{C643}$ cells (Fig. 4C).

\subsection{Apoptosis in C643 Cancer Cells}

Apoptosis may be reckoned as an important obstacle for a damaged cell to become malignant tumors. Since the complexes promote apoptosis induction in cancer cells, flow cytometry using annexin V-FITC / propidium iodide $(\mathrm{PI})$ double staining was carried out for the quantitative discrimination of apoptotic cells. Phosphatidylserine (PS) is a cell cycle signaling phospholipid located inner side of the membrane of a healthy cell but is reverted to the outer membrane for recognition by neighboring cells at the time of apoptosis. Hence, the translocation of phosphatidylserine is a morphological hallmark of apoptosis and can be spotted by its binding with fluorescently labeled annexin $\mathrm{V}$ which in turn detected by flow cytometry. Further the addition of PI to annexin V stained cells is used to discriminate and concomitantly quantify the live cells (lower left quadrant-annexin $\mathrm{V}(-) / \mathrm{PI}(-)$ ), early apoptotic cells (upper left quadrantannexin $\mathrm{V}(+) / \mathrm{PI}(-)$ ) and late apoptotic cells (upper right-quadrant-annexin $\mathrm{V}(+) / \mathrm{PI}(+)$ ) using FACS. As projected in Fig. 4B, the incubation of Gd-PFH-NPs and C-Gd-PFH-NPs with C643 cells conspicuously induced apoptosis. It is worth to note that the titled complexes induce apoptosis even at very low concentrations which is less than their IC50. In comparison with control, the cell population was higher (6-9\%) in annexin V(+)/PI(-) (upper left) quadrant indicating the induction of early apoptosis (Fig. 4D).

This effect was ascertained to be high for C-Gd-PFH-NPs than the Gd-PFH-NPs analogous with the results of MTT, and AO-EB staining assays. It is to note that the test samples displayed comparatively better apoptotic induction on $\mathrm{C643}$ cells.

\subsection{In Vitro Ultrasound Imaging}

Based on C-Gd-PFH-NPs targeted accumulation capacity in tumour cells, we gambled that the phase changing nanoparticles can aid as US contrast to improve USI and treatment scratches. Following the administration of various medicines before LIFUS irradiation, even less or anechoic and less contrast 
improved US signals were noted in each groups (Fig. 5). Six hours after the administration of various treatments, LIFUS was performed in all groups same time periods with in vivo ultrasound imaging. In comparison with the saline, expressively sturdier spot like echo signs slowly accrued in both modes at the tumour places in the treated group, while no evident deviations were detected in the saline group, and only negligible signs looked in the non-target group. This outcome recommended that C225 eased the directing of tumour tissue accretion, and huge quantities of microbubbles were produced when phasechanging NPs were subjected to ADV at the LIFUS triggered tumour site, resultant in improved US imaging. Though, owing to the absence of C225-mediated targeting capacity, the C-Gd-PFH-NPs inadequate ADV could not effectively improve ultrasound imaging. Furthermore, apparent enrichment without LIFUS irradiation was not found in the Gd-PFH-NPs and C-Gd-PFH-NPs alone could not in vitro improve the ultrasound imaging shown in Fig. 5B. These findings showed that because of their relative stability, C-Gd-PFH-NPs were appropriate as ultrasound imaging agents and efficient in vivo nanocarriers.

\subsection{Histological Evaluation for Systemic Toxicity}

The efficiency of anticancer chemotherapeutic drugs is mainly validated by its selective action towards cancer tissues leaving the normal organs undamaged. After the verification of low systemic toxicity in the mice injected with Gd-PFH-NPs (2.5, and $5 \mathrm{mg} / \mathrm{kg}$ ), and C-Gd-PFH-NPs (2.5, and $5 \mathrm{mg} / \mathrm{kg}$ ), histological analyses were carried out to identify the structural changes in the tissues of vital of organs inclusive of heart, liver, spleen, lung, and kidney of the mice treated with Gd-PFH-NPs and C-Gd-PFH-NPs and compared with control, the saline received mice. Figure 6 represented the histological sections of the heart, liver, spleen, lung, and kidney stained with hematoxylin and eosin (H\&E).The photomicrographs of the liver and spleen of the control, Gd-PFH-NPs and C-Gd-PFH-NPs treated groups displayed normal cellular morphology. Under optical microscopy examination, the heart, lung, and kidney of Gd-PFH-NPs and C-Gd-PFH-NPs treated animals showed normal cardiac muscle fibers, normal alveolar, and normal glomerular histological characteristics respectively which were found to be similar histological architecture as those of the control group with no treatment-related inflammatory response.

\subsection{In Vivo Antitumor Efficacy in C643 Xenograft Tumor Model}

Considering the promising in vitro biological activity profiles, the in vivo pharmacological efficacy was further investigated in a 6443 thyriod xenograft tumor model. In the experimental process, body weight of animals in each group was stable. It suggested that the experimental doses in all groups were tolerable. As shown in Fig. 7A-C, we found an obvious retardation of tumor growth for animals treated with Gd-PFHNPs and C-Gd-PFH-NPs, as compared to the control group. Specifically, nanoparticles delivering C-GdPFH-NPs more efficiently suppressed tumor growth than administered Gd-PFH-NPs and saline (Fig. 7) panels a tumor site(s) via the EPR effect. Moreover, these C-Gd-PFH-NPs did not significantly affect the body weights of mice, indicating that the delivery materials and Gd-PFH-NPs have low systemic toxicity. Most importantly, treatment with the combination of C-Gd-PFH-NPs could significantly enhance the 
efficacy of chemotherapy for C-Gd-PFH-NPs, as evidenced by more remarkable slow-down for tumor growth in relative to the Gd-PFH-NPs and saline $(P<0.05)$. On day 33 , animals in saline groups performed a high average tumor weight of $1.58 \mathrm{~g}$ (Fig. 7D). The animals treated with Gd-PFH-NPs and saline exhibited lower mean tumor weight of $0.99 \mathrm{~g}, 0.55 \mathrm{~g}$, and $0.13 \mathrm{~g}$, respectively. A significantly lower mean tumor weight was obvious for C-Gd-PFH-NPs compared to Gd-PFH-NPs and saline $(P<0.05)$. The results of H\&E, TUNEL and Ki67 histopathology analyses were consistent with the results of these therapeutic studies, showing extensive intratumoral apoptosis and reduced cell proliferation caused by the nanoparticle treatments (Fig. 7E). In addition, in H\&E staining of the a C643 thyriod tumor slices, we found extremely aberrant histological structures. Compared with the Gd-PFH-NPs tumor tissues, C-GdPFH-NPs tumors presented more abundant extracellular matrix with messier cell distribution, which closely recapitulates tumors in thyriod cancer patients.

\section{Conclusion}

The data offered here highpoint a strategy rationale for concurrently attractive the effectiveness and safety of extremely Gd-PFA-NPs. As the synthetic Gd-PFA-NPs and C-Gd-PFA-NPs are fully biocompatible composites with minimal modifications, the safety risks can be minimized when considering their clinical translation. Furthermore, given the ability of Gd-PFA-NPs to overcome the Cetuximab (C225)-Conjugated C-Gd-PFA-NPs, it was expected that our approach could have high value as an optional therapeutic platform to treat patients with drug-resistant cancer. Lastly, we envision that in addition to taxane agents, this C-Gd-PFA-NPs -based approach could be a simple yet broadly applicable strategy to make improved tolerated and more well-organized cytotoxic nanotherapeutics from other antitumor agents.

\section{Declarations}

\section{Competing interests}

The authors declare that they have no competing interests.

\section{References}

[1] L. Zhu, H. Zhao, Z. Zhou, Y. Xia, Z. Wang, H. Ran, P. Li, J. Ren, Peptide-Functionalized PhaseTransformation Nanoparticles for Low Intensity Focused Ultrasound-Assisted Tumor Imaging and Therapy, Nano Letters. 18 (2018) 1831-1841. doi:10.1021/acs.nanolett.7b05087.

[2] Q. Zhou, Y. Zeng, Q. Xiong, S. Zhong, P. Li, H. Ran, Y. Yin, C. Reutelingsperger, F.W. Prinze, Z. Ling, Construction of CNA35 Collagen-Targeted Phase-Changeable Nanoagents for Low-Intensity Focused Ultrasound-Triggered Ultrasound Molecular Imaging of Myocardial Fibrosis in Rabbits, ACS Applied Materials \& Interfaces. 11 (2019) 23006-23017. doi:10.1021/acsami.9b05999. 
[3] M. Wang, Q. Yang, M. Li, H. Zou, Z. Wang, H. Ran, Y. Zheng, J. Jian, Y. Zhou, Y. Luo, Y. Ran, S. Jiang, X. Zhou, Multifunctional Nanoparticles for Multimodal Imaging-Guided Low-Intensity Focused Ultrasound/Immunosynergistic Retinoblastoma Therapy, ACS Applied Materials \& Interfaces. 12 (2020) 5642-5657. doi:10.1021/acsami.9b22072.

[4] Y. Zhong, Y. Zhang, J. Xu, J. Zhou, J. Liu, M. Ye, L. Zhang, B. Qiao, Z. Wang, H. Ran, D. Guo, LowIntensity Focused Ultrasound-Responsive Phase-Transitional Nanoparticles for Thrombolysis without Vascular Damage: A Synergistic Nonpharmaceutical Strategy, ACS Nano. 13 (2019) 3387-3403. doi:10.1021/acsnano.8b09277.

[5] N.A. Tokranova, S.W. Novak, J. Castracane, I.A. Levitsky, Deep Infiltration of Emissive Polymers into Mesoporous Silicon Microcavities: Nanoscale Confinement and Advanced Vapor Sensing, The Journal of Physical Chemistry C. 117 (2013) 22667-22676. doi:10.1021/jp405071n.

[6] J. Liu, F. Xu, J. Huang, J. Xu, Y. Liu, Y. Yao, M. Ao, A. Li, L. Hao, Y. Cao, Z. Hu, H. Ran, Z. Wang, P. Li, Lowintensity focused ultrasound (LIFU)-activated nanodroplets as a theranostic agent for noninvasive cancer molecular imaging and drug delivery, Biomaterials Science. 6 (2018) 2838-2849.

doi:10.1039/C8BM00726H.

[7] A. Yang, B. Qiao, E.M. Strohm, J. Cao, Z. Wang, X. Yuan, Y. Luo, Y. Sun, Thrombin-responsive engineered nanoexcavator with full-thickness infiltration capability for pharmaceutical-free deep venous thrombosis theranostics, Biomaterials Science. 8 (2020) 4545-4558. doi:10.1039/D0BM00917B.

[8] Y. Wang, G. Sui, D. Teng, Q. Wang, J. Qu, L. Zhu, H. Ran, Z. Wang, C. Jin, H. Wang, Low intensity focused ultrasound (LIFU) triggered drug release from cetuximab-conjugated phase-changeable nanoparticles for precision theranostics against anaplastic thyroid carcinoma, Biomaterials Science. 7 (2019) 196-210. doi:10.1039/C8BM00970H.

[9] T. Iwashina, J. Mochida, T. Miyazaki, T. Watanabe, S. Iwabuchi, K. Ando, T. Hotta, D. Sakai, Lowintensity pulsed ultrasound stimulates cell proliferation and proteoglycan production in rabbit intervertebral disc cells cultured in alginate, Biomaterials. 27 (2006) 354-361.

doi:https://doi.org/10.1016/j.biomaterials.2005.06.031.

[10] Y. Kang, J. Kim, J. Park, Y.M. Lee, G. Saravanakumar, K.M. Park, W. Choi, K. Kim, E. Lee, C. Kim, W.J. Kim, Tumor vasodilation by $\mathrm{N}$-Heterocyclic carbene-based nitric oxide delivery triggered by high-intensity focused ultrasound and enhanced drug homing to tumor sites for anti-cancer therapy, Biomaterials. 217 (2019) 119297. doi:https://doi.org/10.1016/j.biomaterials.2019.119297.

[11] Y.-J. Ho, C.-H. Wu, Q. Jin, C.-Y. Lin, P.-H. Chiang, N. Wu, C.-H. Fan, C.-M. Yang, C.-K. Yeh, Superhydrophobic drug-loaded mesoporous silica nanoparticles capped with $\beta$-cyclodextrin for ultrasound image-guided combined antivascular and chemo-sonodynamic therapy, Biomaterials. 232 (2020) 119723. doi:https://doi.org/10.1016/j.biomaterials.2019.119723. 
[12] Y. Wang, J. Zhang, X. Lv, L. Wang, Z. Zhong, D.-P. Yang, W. Si, T. Zhang, X. Dong, Mitoxantrone as photothermal agents for ultrasound/fluorescence imaging-guided chemo-phototherapy enhanced by intratumoral H2O2-Induced CO, Biomaterials. 252 (2020) 120111. doi:https://doi.org/10.1016/j.biomaterials.2020.120111.

[13] J. Pan, L. Zhang, S. Xu, X. Cheng, H. Yu, J. Bao, R. Lu, Induction of Apoptosis in Human PapillaryThyroid-Carcinoma BCPAP Cells by Diallyl Trisulfide through Activation of the MAPK Signaling Pathway, Journal of Agricultural and Food Chemistry. 66 (2018) 5871-5878. doi:10.1021/acs.jafc.8b02243.

[14] A. Cutignano, B. Seetharamsingh, D. D’Angelo, G. Nuzzo, P. V Khairnar, A. Fusco, D.S. Reddy, A. Fontana, Identification and Synthesis of Mycalol Analogues with Improved Potency against Anaplastic Thyroid Carcinoma Cell Lines, Journal of Natural Products. 80 (2017) 1125-1133. doi:10.1021/acs.jnatprod.7b00043.

[15] D. Paolino, D. Cosco, M. Licciardi, G. Giammona, M. Fresta, G. Cavallaro, Polyaspartylhydrazide Copolymer-Based Supramolecular Vesicular Aggregates as Delivery Devices for Anticancer Drugs, Biomacromolecules. 9 (2008) 1117-1130. doi:10.1021/bm700964a.

[16] J. Zheng, X. Cheng, S. Xu, L. Zhang, J. Pan, H. Yu, J. Bao, R. Lu, Diallyl trisulfide induces G2/M cellcycle arrest and apoptosis in anaplastic thyroid carcinoma 8505C cells, Food \& Function. 10 (2019) 7253-7261. doi:10.1039/C9F000646J.

[17] A. Balbín, F. Gaballo, J. Ceballos-Torres, S. Prashar, M. Fajardo, G.N. Kaluđerović, S. Gómez-Ruiz, Dual application of Pd nanoparticles supported on mesoporous silica SBA-15 and MSU-2: supported catalysts for $\mathrm{C}-\mathrm{C}$ coupling reactions and cytotoxic agents against human cancer cell lines, RSC Advances. 4 (2014) 54775-54787. doi:10.1039/C4RA11759J.

[18] J.P. Parker, Z. Ude, C.J. Marmion, Exploiting developments in nanotechnology for the preferential delivery of platinum-based anti-cancer agents to tumours: targeting some of the hallmarks of cancer, Metallomics. 8 (2016) 43-60. doi:10.1039/C5MT00181A.

[19] M. Zhou, Y. Chen, M. Adachi, X. Wen, B. Erwin, O. Mawlawi, S.Y. Lai, C. Li, Single agent nanoparticle for radiotherapy and radio-photothermal therapy in anaplastic thyroid cancer, Biomaterials. 57 (2015) 41-49. doi:https://doi.org/10.1016/j.biomaterials.2015.04.013.

[20] X. Kong, P. Pan, H. Sun, H. Xia, X. Wang, Y. Li, T. Hou, Drug Discovery Targeting Anaplastic Lymphoma Kinase (ALK), Journal of Medicinal Chemistry. 62 (2019) 10927-10954.

doi:10.1021/acs.jmedchem.9b00446.

[21] L. Lee, L.M. Robb, M. Lee, R. Davis, H. Mackay, S. Chavda, B. Babu, E.L. O'Brien, A.L. Risinger, S.L. Mooberry, M. Lee, Design, Synthesis, and Biological Evaluations of 2,5-Diaryl-2,3-dihydro-1,3,4oxadiazoline Analogs of Combretastatin-A4, Journal of Medicinal Chemistry. 53 (2010) 325-334. doi:10.1021/jm901268n. 
[22] A. Arcinas, T.-Y. Yen, E. Kebebew, B.A. Macher, Cell Surface and Secreted Protein Profiles of Human Thyroid Cancer Cell Lines Reveal Distinct Glycoprotein Patterns, Journal of Proteome Research. 8 (2009) 3958-3968. doi:10.1021/pr900278c.

[23] Y. Hu, X.-Z. Dong, X. Liu, P. Liu, Y.-B. Chen, Enhanced Antitumor Activity of Cetuximab in Combination with the Jak Inhibitor CYT387 against Non-Small-Cell Lung Cancer with Various Genotypes, Molecular Pharmaceutics. 13 (2016) 689-697. doi:10.1021/acs.molpharmaceut.5b00927.

[24] D. Zeng, Y. Guo, A.G. White, Z. Cai, J. Modi, R. Ferdani, C.J. Anderson, Comparison of Conjugation Strategies of Cross-Bridged Macrocyclic Chelators with Cetuximab for Copper-64 Radiolabeling and PET Imaging of EGFR in Colorectal Tumor-Bearing Mice, Molecular Pharmaceutics. 11 (2014) 3980-3987. doi: $10.1021 / \mathrm{mp} 500004 \mathrm{~m}$.

[25] S. Heskamp, O.C. Boerman, J.D.M. Molkenboer-Kuenen, F.C.G.J. Sweep, A. Geurts-Moespot, M.S. Engelhardt, W.T.A. van der Graaf, W.J.G. Oyen, H.W.M. van Laarhoven, Cetuximab Reduces the Accumulation of Radiolabeled Bevacizumab in Cancer Xenografts without Decreasing VEGF Expression, Molecular Pharmaceutics. 11 (2014) 4249-4257. doi:10.1021/mp500460g.

[26] F.L. Sung, R.T.K. Pang, B.B.Y. Ma, M.M.L. Lee, S.M. Chow, T.C.W. Poon, A.T.C. Chan, Pharmacoproteomics Study of Cetuximab in Nasopharyngeal Carcinoma, Journal of Proteome Research. 5 (2006) 3260-3267. doi:10.1021/pr050452g.

[27] H. Kim, S.-H. Goh, Y. Choi, Quenched cetuximab conjugate for fast fluorescence imaging of EGFRpositive lung cancers, Biomaterials Science. (2020). doi:10.1039/D0BM01148G.

[28] D.-H. Kim, D.-K. Kim, K. Zhou, S. Park, Y. Kwon, M.G. Jeong, N.K. Lee, S.H. Ryu, Single particle trackingbased reaction progress kinetic analysis reveals a series of molecular mechanisms of cetuximab-induced EGFR processes in a single living cell, Chemical Science. 8 (2017) 4823-4832.

doi:10.1039/C7SC01159H.

[29] L. García-Fernández, J. Garcia-Pardo, O. Tort, I. Prior, M. Brust, E. Casals, J. Lorenzo, V.F. Puntes, Conserved effects and altered trafficking of Cetuximab antibodies conjugated to gold nanoparticles with precise control of their number and orientation, Nanoscale. 9 (2017) 6111-6121. doi:10.1039/C7NR00947J.

[30] J. Kim, S.Y. Woo, C.Y. Im, E.K. Yoo, S. Lee, H.-J. Kim, H.-J. Hwang, J. Cho, W.S. Lee, H. Yoon, S. Kim, O. Kwon, H. Hwang, K.-H. Kim, J.-H. Jeon, T.D. Singh, S.W. Kim, S.Y. Hwang, H.-S. Choi, I.-K. Lee, S.H. Kim, Y.H. Jeon, J. Chin, S.J. Cho, Insights of a Lead Optimization Study and Biological Evaluation of Novel 4Hydroxytamoxifen Analogs as Estrogen-Related Receptor Y (ERRY) Inverse Agonists, Journal of Medicinal Chemistry. 59 (2016) 10209-10227. doi:10.1021/acs.jmedchem.6b01204.

[31] J. Kim, J. Song, H.D. Ji, E.K. Yoo, J.-E. Lee, S.B. Lee, J.M. Oh, S. Lee, J.S. Hwang, H. Yoon, D.-S. Kim, S.J. Lee, M. Jeong, S. Lee, K.-H. Kim, H.-S. Choi, S.W. Lee, K.-G. Park, I.-K. Lee, S.H. Kim, H. Hwang, Y.H. Jeon, 
J. Chin, S.J. Cho, Discovery of Potent, Selective, and Orally Bioavailable Estrogen-Related Receptor-y Inverse Agonists To Restore the Sodium lodide Symporter Function in Anaplastic Thyroid Cancer, Journal of Medicinal Chemistry. 62 (2019) 1837-1858. doi:10.1021/acs.jmedchem.8b01296.

[32] R. Suriano, Y. Lin, B.T. Ashok, S.D. Schaefer, S.P. Schantz, J. Geliebter, R.K. Tiwari, Pilot Study Using SELDI-TOF-MS Based Proteomic Profile for the Identification of Diagnostic Biomarkers of Thyroid Proliferative Diseases, Journal of Proteome Research. 5 (2006) 856-861. doi:10.1021/pr050349r.

[33] W.-C. Wang, H.-Y. Shiao, C.-C. Lee, K.-S. Fung, H.-P. Hsieh, Anaplastic lymphoma kinase (ALK) inhibitors: a review of design and discovery, MedChemComm. 5 (2014) 1266-1279. doi:10.1039/C4MD00048J.

[34] 0.I. Parisi, C. Morelli, L. Scrivano, M.S. Sinicropi, M.G. Cesario, S. Candamano, F. Puoci, D. Sisci, Controlled release of sunitinib in targeted cancer therapy: smart magnetically responsive hydrogels as restricted access materials, RSC Advances. 5 (2015) 65308-65315. doi:10.1039/C5RA12229E.

[35] L. Naso, M. Valcarcel, P. Villacé, M. Roura-Ferrer, C. Salado, E.G. Ferrer, P.A.M. Williams, Specific antitumor activities of natural and oxovanadium(iv) complexed flavonoids in human breast cancer cells, New Journal of Chemistry. 38 (2014) 2414-2421. doi:10.1039/C3NJ01649H.

[36] B.P. Nair, C.P. Sharma, Poly(lactide-co-glycolide)-Laponite-F68 Nanocomposite Vesicles through a Single-Step Double-Emulsion Method for the Controlled Release of Doxorubicin, Langmuir. 28 (2012) 4559-4564. doi:10.1021/la300005c.

[37] K. Kamiya, S. Takeuchi, Giant liposome formation toward the synthesis of well-defined artificial cells, Journal of Materials Chemistry B. 5 (2017) 5911-5923. doi:10.1039/C7TB01322A.

[38] M. Nabi-Meibodi, A. Vatanara, A.R. Najafabadi, M.R. Rouini, V. Ramezani, K. Gilani, S.M.H. Etemadzadeh, K. Azadmanesh, The effective encapsulation of a hydrophobic lipid-insoluble drug in solid lipid nanoparticles using a modified double emulsion solvent evaporation method, Colloids and Surfaces B: Biointerfaces. 112 (2013) 408-414. doi:https://doi.org/10.1016/j.colsurfb.2013.06.013.

[39] A.Z.M. Badruddoza, S. V MacWilliams, D.A. Sebben, M. Krasowska, D. Beattie, D.J. Durian, J.K. Ferri, Diffusing wave spectroscopy (DWS) methods applied to double emulsions, Current Opinion in Colloid \& Interface Science. 37 (2018) 74-87. doi:https://doi.org/10.1016/j.cocis.2018.06.006.

[40] L. Cai, X. Qin, Z. Xu, Y. Song, H. Jiang, Y. Wu, H. Ruan, J. Chen, Comparison of Cytotoxicity Evaluation of Anticancer Drugs between Real-Time Cell Analysis and CCK-8 Method, ACS Omega. 4 (2019) 1203612042. doi:10.1021/acsomega.9b01142.

[41] G. Jiao, X. He, X. Li, J. Qiu, H. Xu, N. Zhang, S. Liu, Limitations of MTT and CCK-8 assay for evaluation of graphene cytotoxicity, RSC Advances. 5 (2015) 53240-53244. doi:10.1039/C5RA08958A. 
[42] H.X. Ren, Q.C. Tang, L. Yan, H. Xia, H.S. Luo, Evodiamine inhibits gastrointestinal motility via CCK and CCK1 receptor in water-avoidence stress rat model, Life Sciences. 209 (2018) 210-216.

doi:https://doi.org/10.1016/j.lfs.2018.08.003.

[43] S. Kasibhatla, G.P. Amarante-Mendes, D. Finucane, T. Brunner, E. Bossy-Wetzel, D.R. Green, Acridine Orange/Ethidium Bromide (AO/EB) Staining to Detect Apoptosis, CSH Protocols. 2006 (2006) 799-803. doi:10.1101/pdb.prot4493.

[44] K. Liu, P. Liu, R. Liu, X. Wu, Dual AO/EB staining to detect apoptosis in osteosarcoma cells compared with flow cytometry, Medical Science Monitor Basic Research. 21 (2015) 15-20. doi:10.12659/MSMBR.893327.

[45] W.-H. Zhang, S. Chen, X.-L. Liu, Bing-Lin, X.-W. Liu, Y. Zhou, Study on antitumor activities of the chrysin-chromene-spirooxindole on Lewis lung carcinoma C57BL/ 6 mice in vivo, Bioorganic \& Medicinal Chemistry Letters. 30 (2020) 127410. doi:https://doi.org/10.1016/j.bmcl.2020.127410.

[46] T.E. Yalcin, S. Ilbasmis-Tamer, S. Takka, Antitumor activity of gemcitabine hydrochloride loaded lipid polymer hybrid nanoparticles (LPHNs): In vitro and in vivo, International Journal of Pharmaceutics. 580 (2020) 119246. doi:https://doi.org/10.1016/j.ijpharm.2020.119246.

[47] F.A. Kabeer, D.S. Rajalekshmi, M.S. Nair, R. Prathapan, In vitro and in vivo antitumor activity of deoxyelephantopin from a potential medicinal plant Elephantopus scaber against Ehrlich ascites carcinoma, Biocatalysis and Agricultural Biotechnology. 19 (2019) 101106.

doi:https://doi.org/10.1016/j.bcab.2019.101106.

\section{Figures}




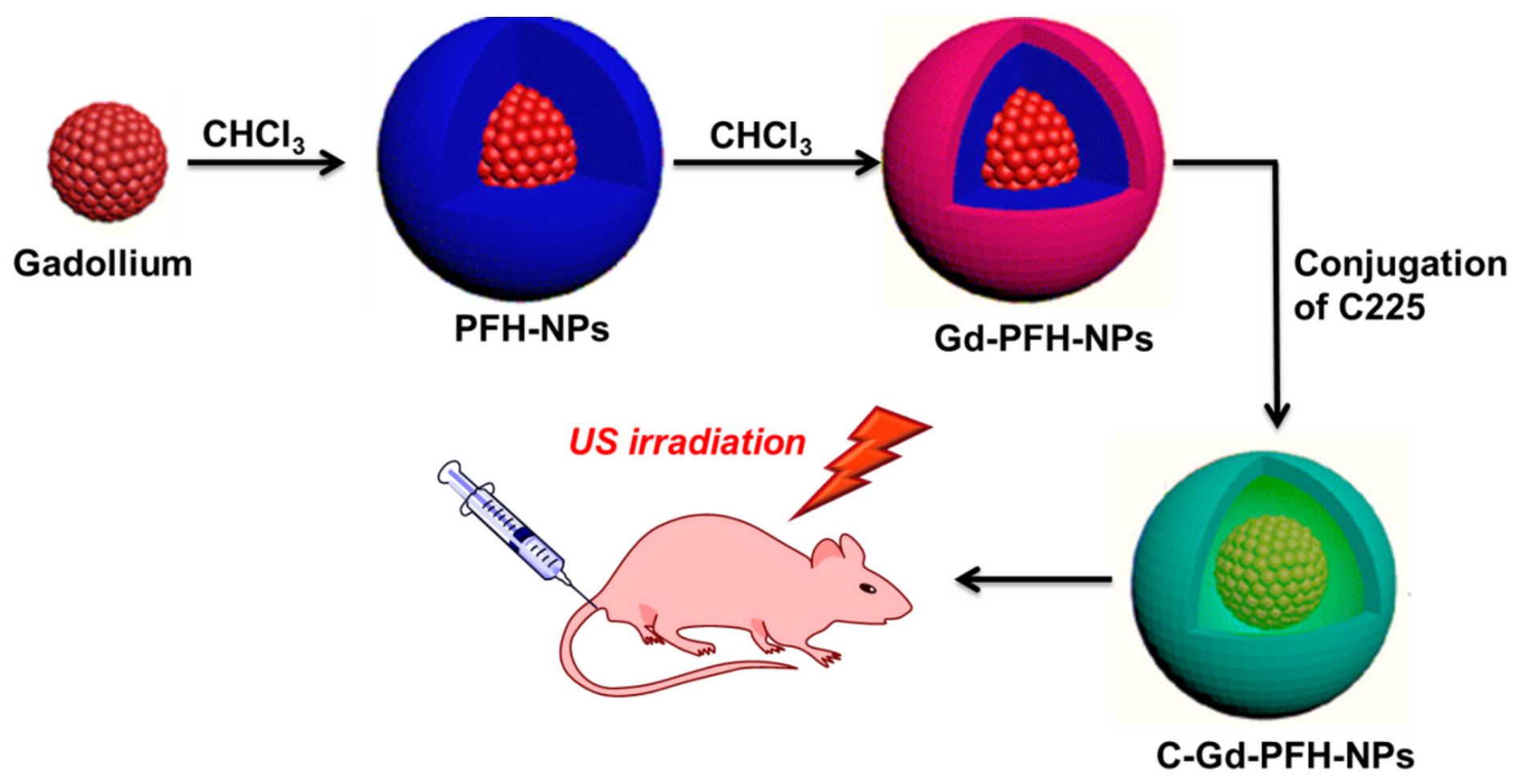

Figure 1

Schematic illustration of the microstructure of C-Gd-PFH-NPs and the phase-transformation process by means of LIFUS ultrasound irradiation. Meanwhile, a schematic of LIFUS ablation principles.

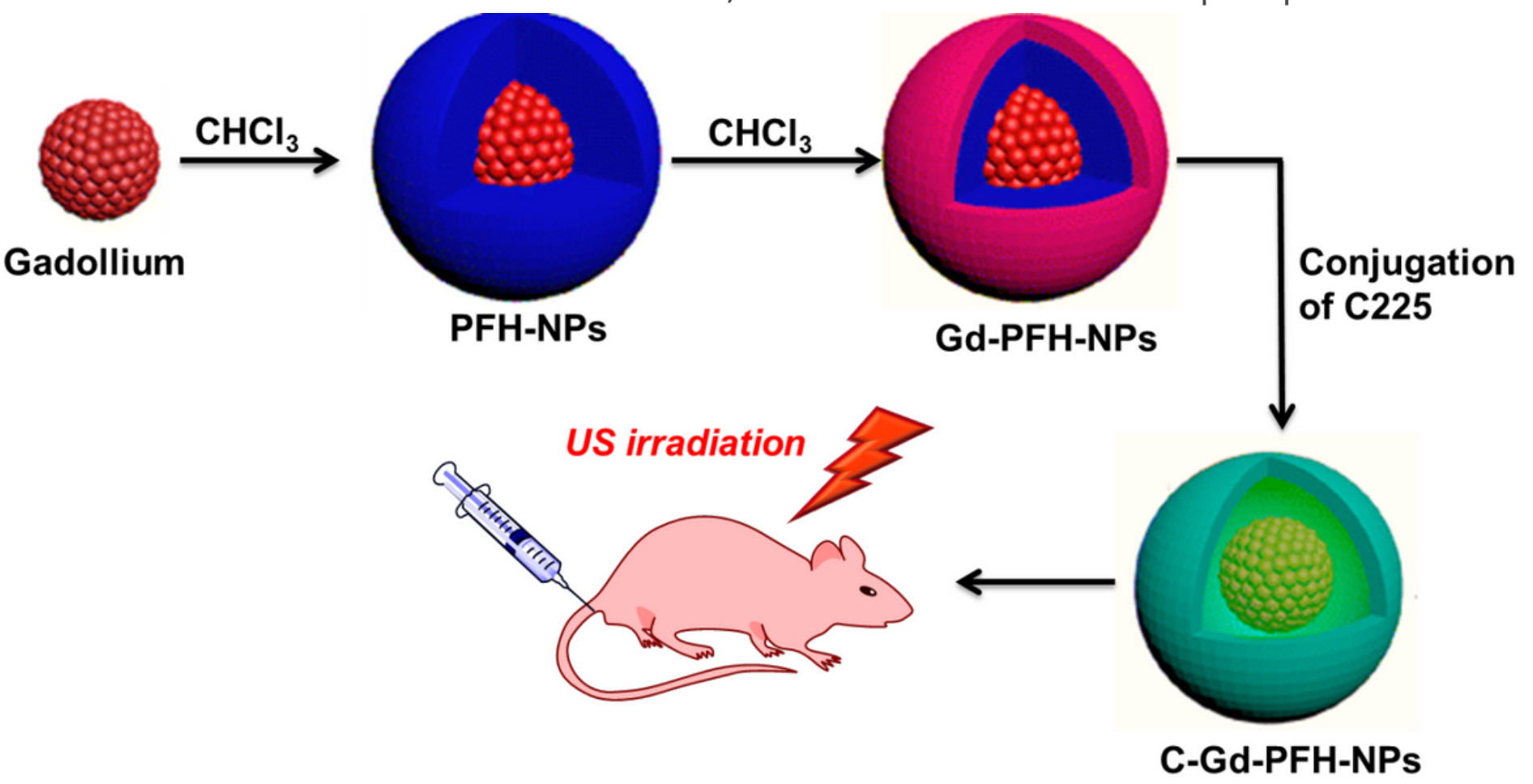

Figure 1 
Schematic illustration of the microstructure of C-Gd-PFH-NPs and the phase-transformation process by means of LIFUS ultrasound irradiation. Meanwhile, a schematic of LIFUS ablation principles.

A

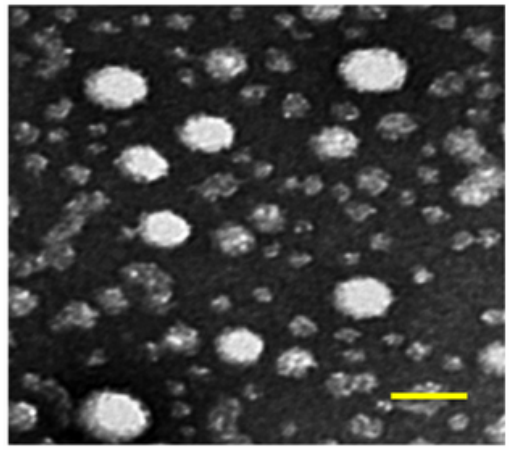

B

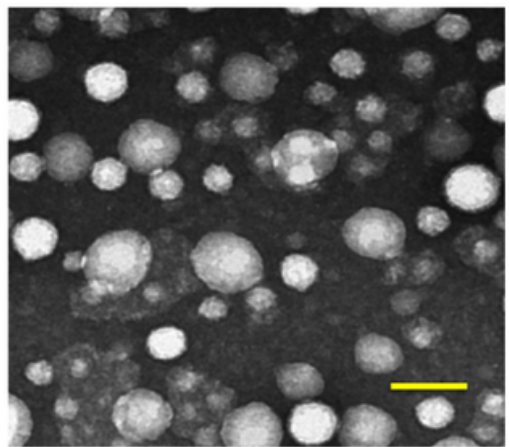

C

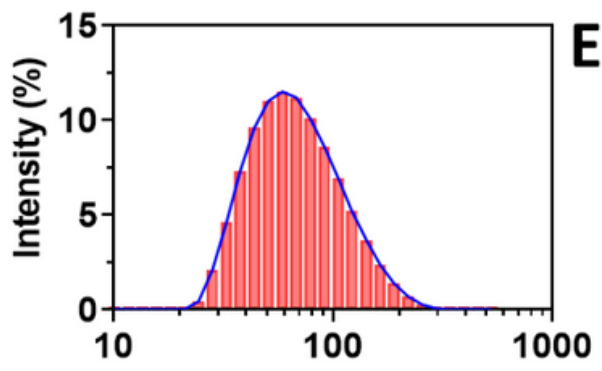

Particle size (nm)

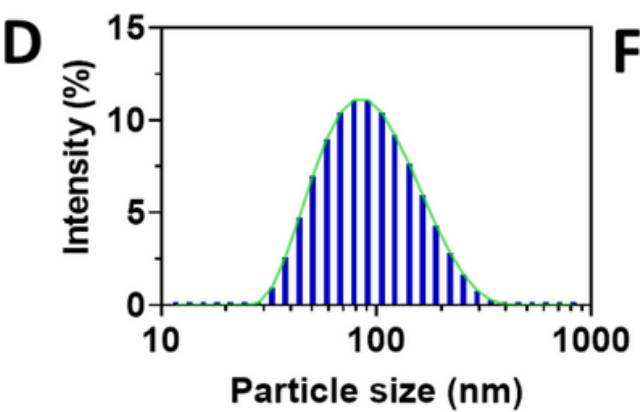

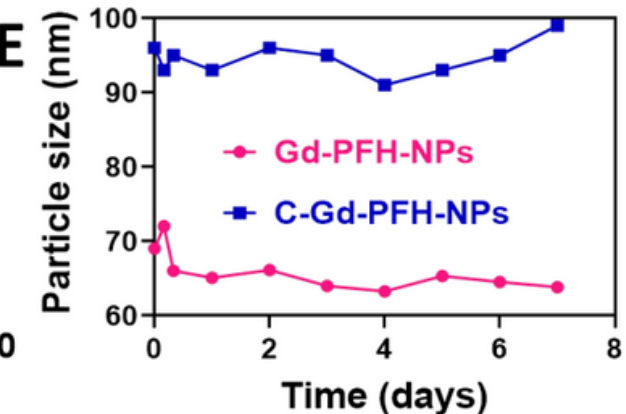

$\mathbf{F}$

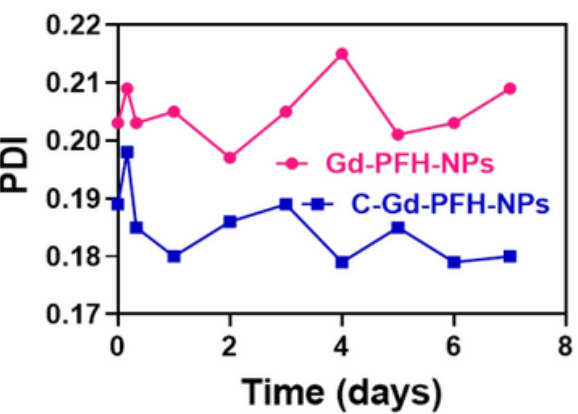

Figure 2

A) TEM image of Gd-PFH-NPs and B) C-Gd-PFH-NPs. Scale bars, $100 \mathrm{~nm}$ (B and D) DLS image of GdPFH-NPs and C-Gd-PFH-NPs. (E and F) Stability of Gd-PFH-NPs and C-Gd-PFH-NPs. in water with PBS at $37^{\circ} \mathrm{C}$.

A

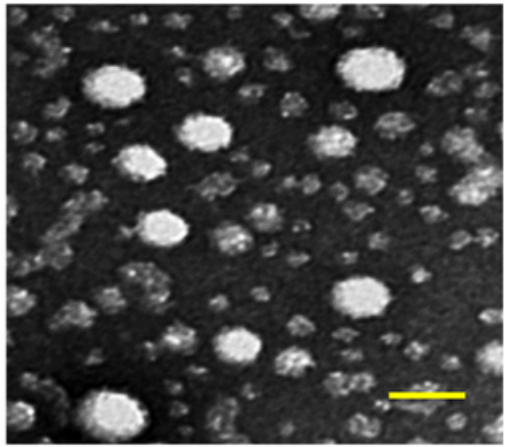

B

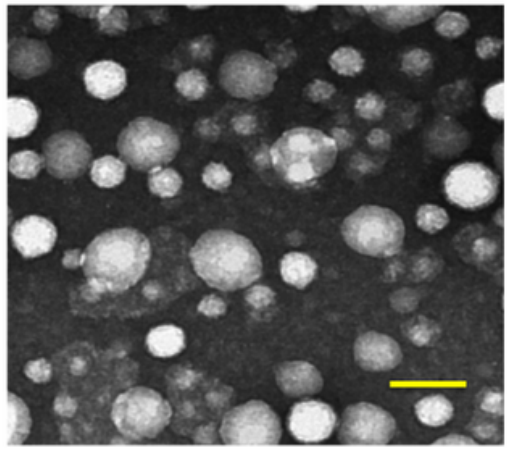

C

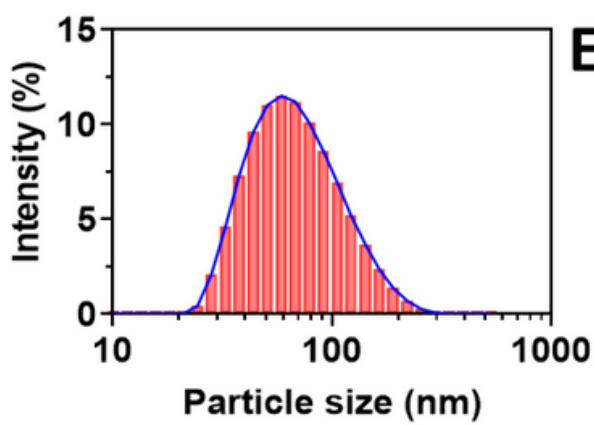

Particle size (nm)

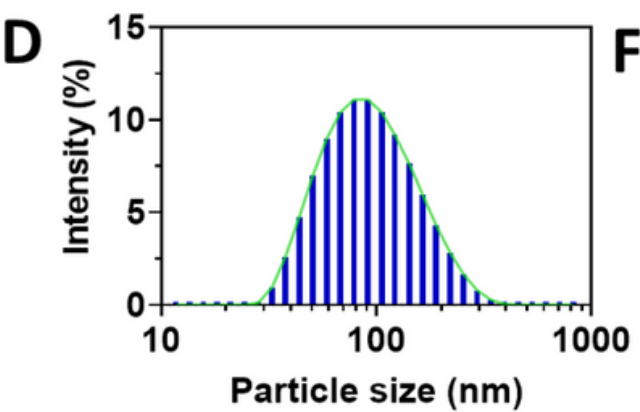

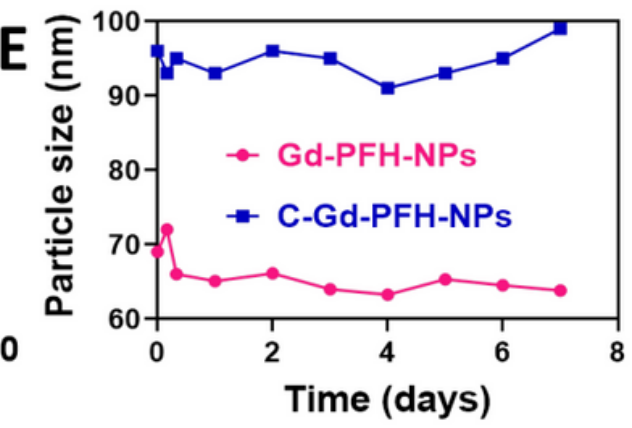

F

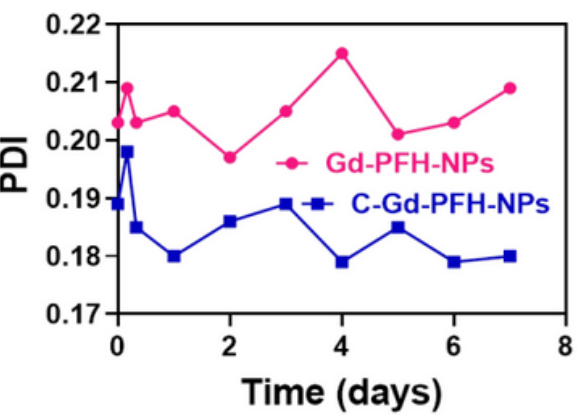

Figure 2 
A) TEM image of Gd-PFH-NPs and B) C-Gd-PFH-NPs. Scale bars, $100 \mathrm{~nm}$ (B and D) DLS image of GdPFH-NPs and C-Gd-PFH-NPs. (E and F) Stability of Gd-PFH-NPs and C-Gd-PFH-NPs. in water with PBS at $37^{\circ} \mathrm{C}$.

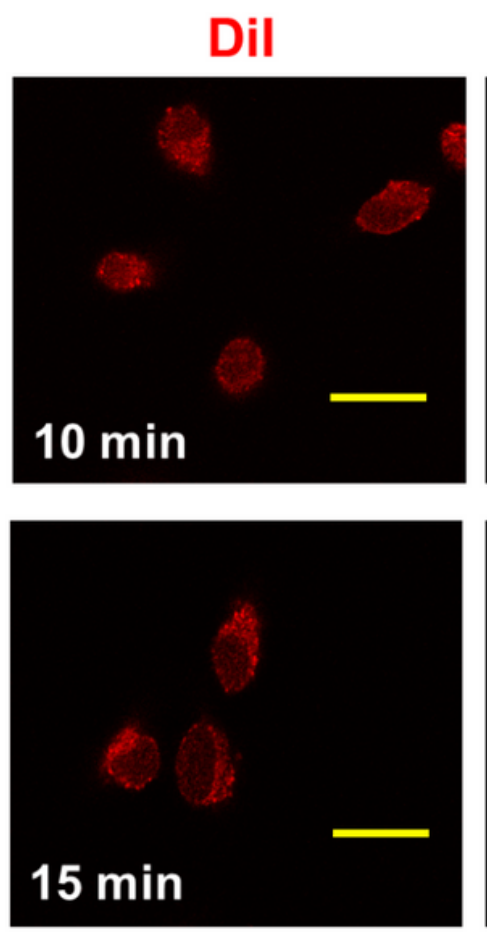

Lysotracker
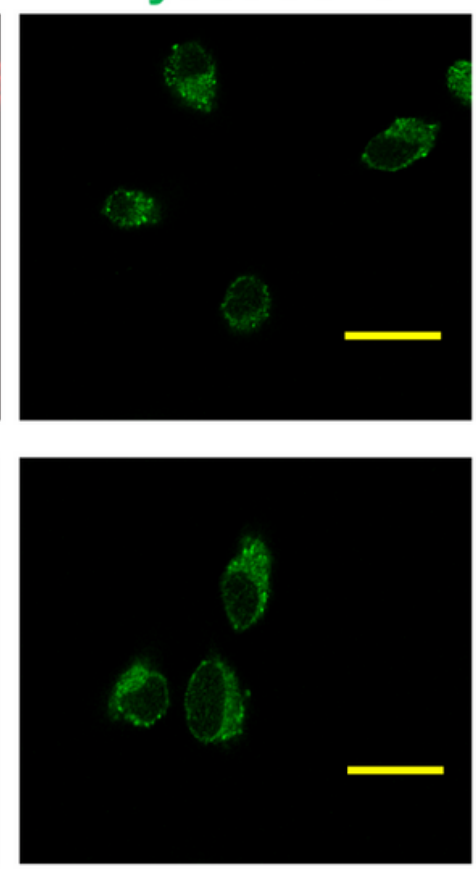

DAPI
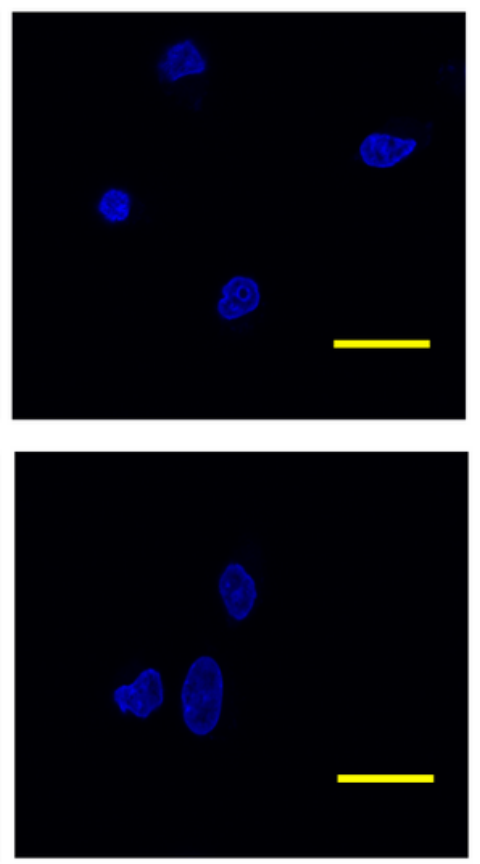

Merge
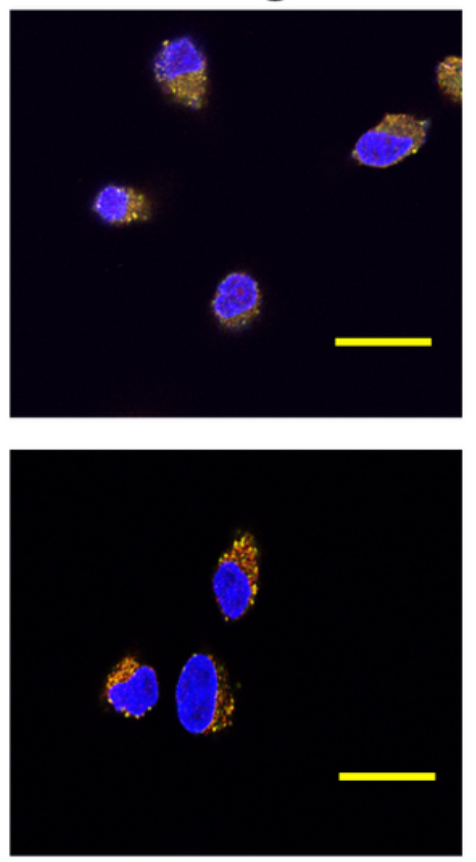

Figure 3

Cellular uptake of C-Gd-PFH-NPs with 10 min and 15 minutes interval.

\section{Dil}
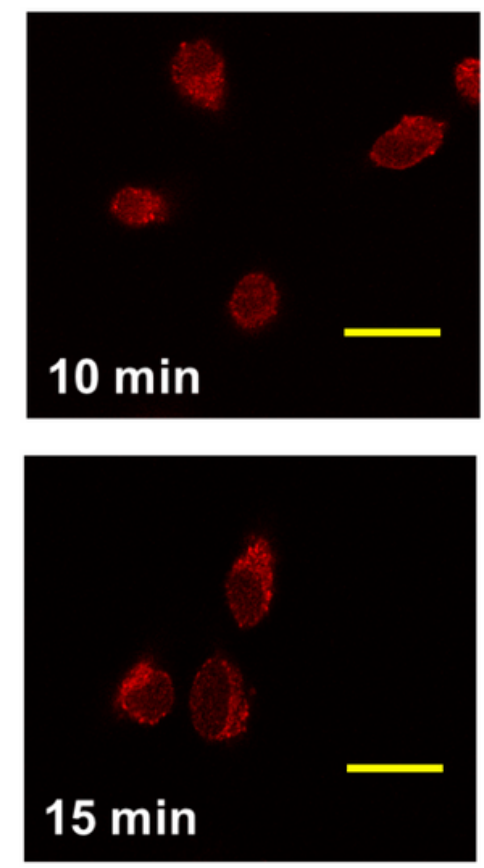

Lysotracker
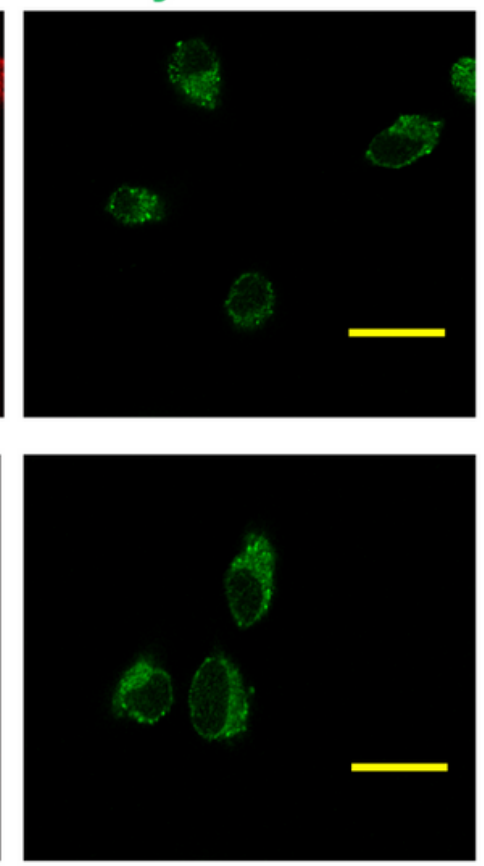

DAPI
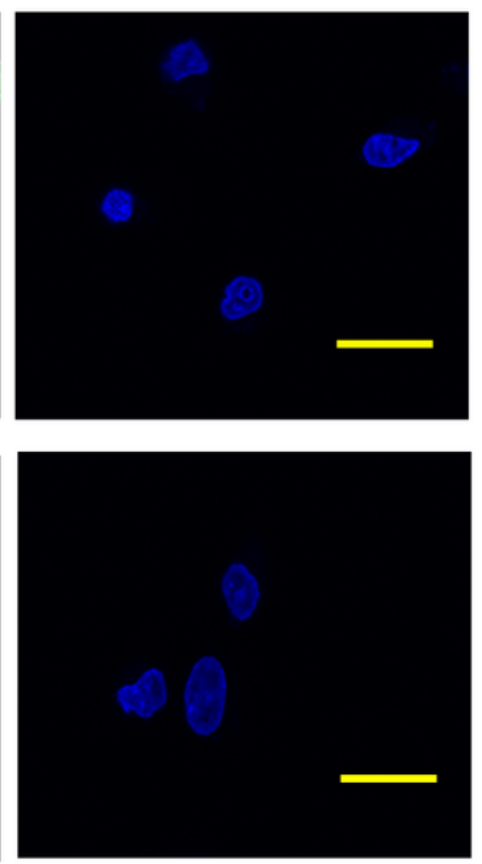

Merge
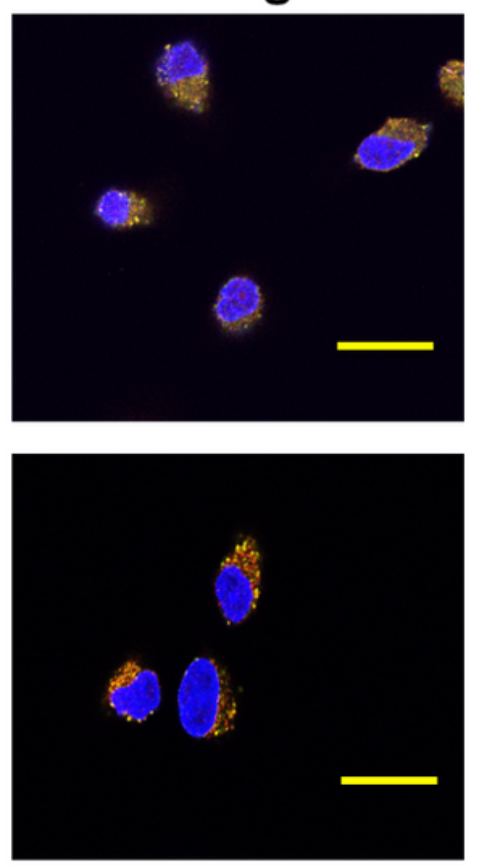
Figure 3

Cellular uptake of C-Gd-PFH-NPs with 10 min and 15 minutes interval.
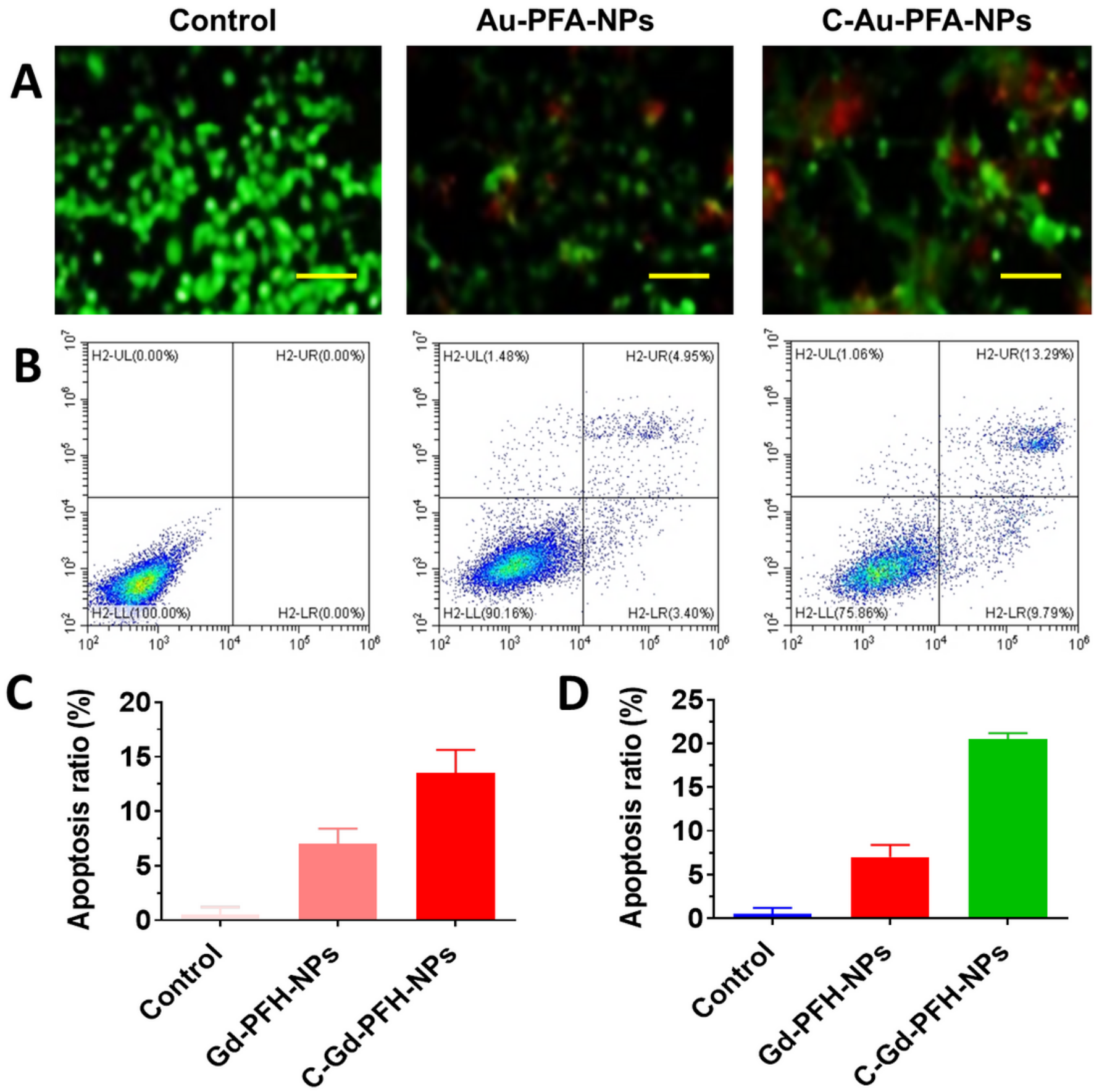

Figure 4

A) Dual AO/EB staining assay for examining Gd-PFH-NPs and C-Gd-PFH-NPs -induced cell death in C643 cells. The cells were treated with Gd-PFH-NPs and C-Gd-PFH-NPs at $2.5 \mu \mathrm{M}$ concentration for $24 \mathrm{~h}$. B) Quantification of apoptosis ratio. The cells were quantified by image J software. C) Apoptotic analysis of C643 cells using flow cytometry. The cells were treated with Gd-PFH-NPs and C-Gd-PFH-NPs at $2.5 \mu \mathrm{M}$ 
concentration for $24 \mathrm{~h}$ and then stained with FITC annexin V/PI for flow cytometry analysis. D) Apoptosis ratio of $\mathrm{C} 643$ cells.
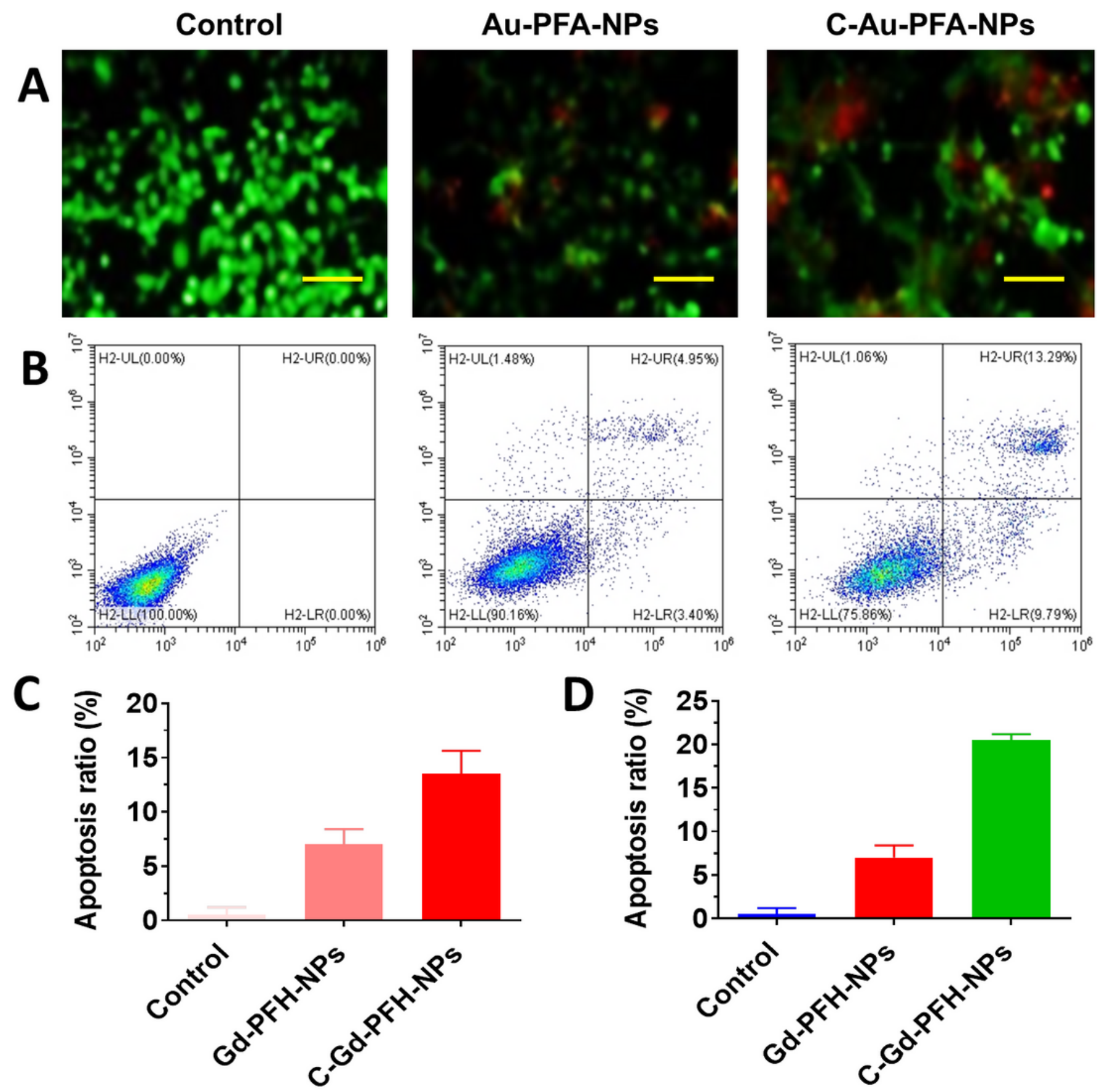

Figure 4

A) Dual AO/EB staining assay for examining Gd-PFH-NPs and C-Gd-PFH-NPs -induced cell death in C643 cells. The cells were treated with Gd-PFH-NPs and C-Gd-PFH-NPs at $2.5 \mu \mathrm{M}$ concentration for $24 \mathrm{~h}$. B) Quantification of apoptosis ratio. The cells were quantified by image $\mathrm{J}$ software. C) Apoptotic analysis of C643 cells using flow cytometry. The cells were treated with Gd-PFH-NPs and C-Gd-PFH-NPs at $2.5 \mu \mathrm{M}$ 
concentration for $24 \mathrm{~h}$ and then stained with FITC annexin V/PI for flow cytometry analysis. D) Apoptosis ratio of $\mathrm{C} 643$ cells.

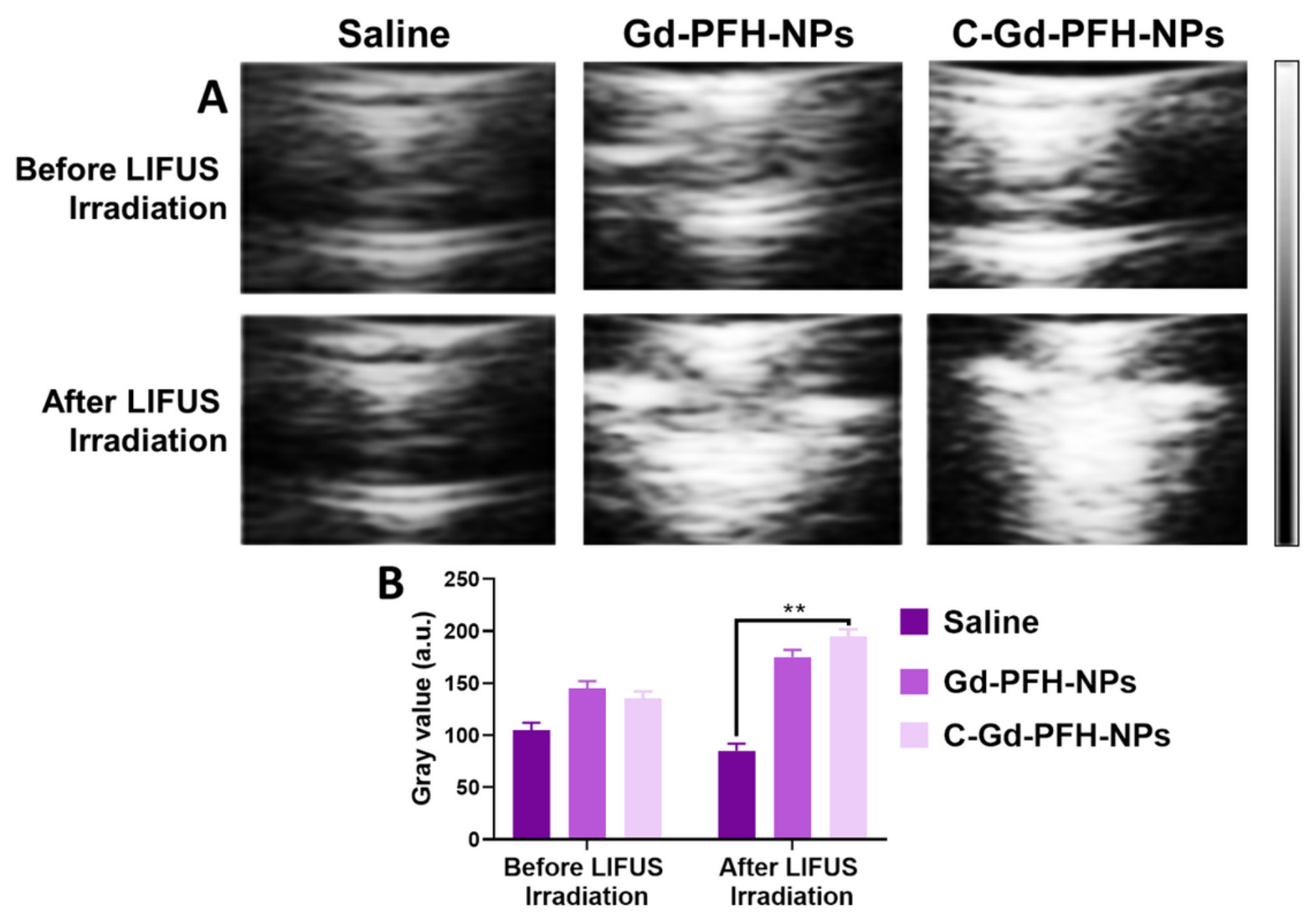

Figure 5

A) In vitro B-mode US imaging of Saline, Gd-PFH-NPs, and C-Gd-PFH-NPs before and after NIR laser irradiation. B) Gray values of B-mode US imaging of Saline, Gd-PFH-NPs, and C-Gd-PFH-NPs before and after NIR laser irradiation. $* * \mathrm{P}<0.01$. 


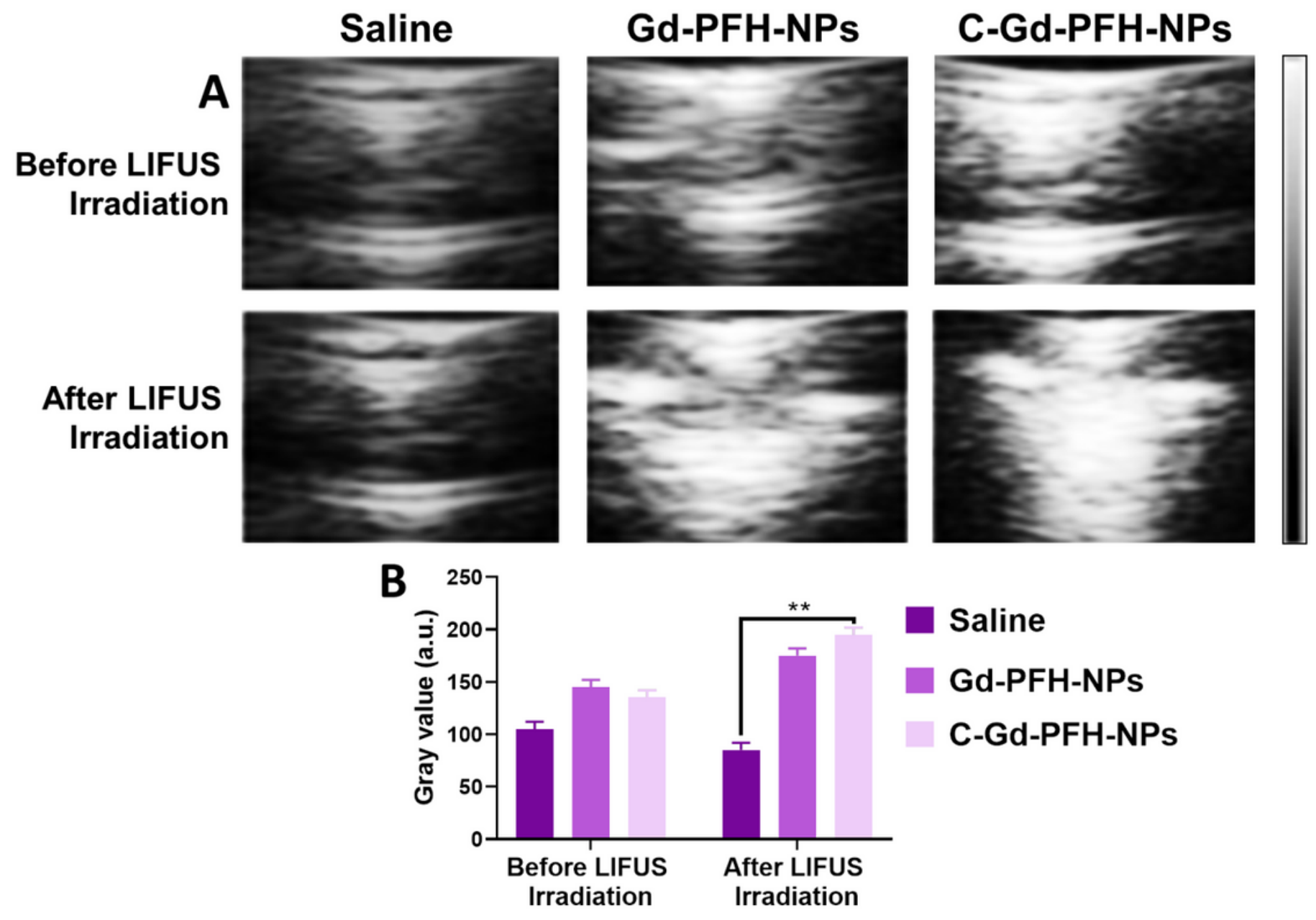

Figure 5

A) In vitro B-mode US imaging of Saline, Gd-PFH-NPs, and C-Gd-PFH-NPs before and after NIR laser irradiation. B) Gray values of B-mode US imaging of Saline, Gd-PFH-NPs, and C-Gd-PFH-NPs before and after NIR laser irradiation. ${ }^{\star \star} \mathrm{P}<0.01$. 


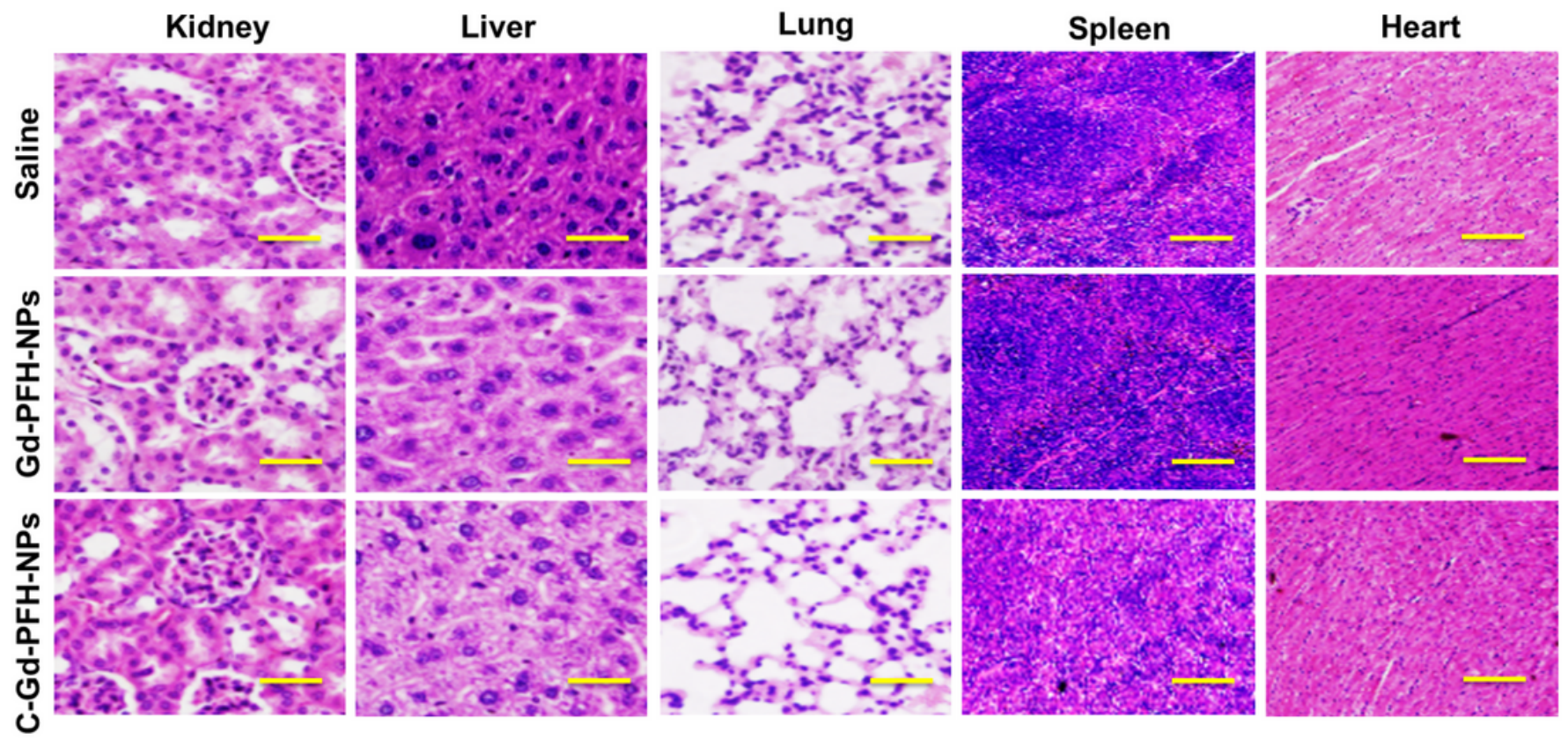

Figure 6

H\&E staining of the major organs (kidney, liver, lung, spleen and heart) excised from different treatment mice groups. Scale bar: $100 \mu \mathrm{m}$.
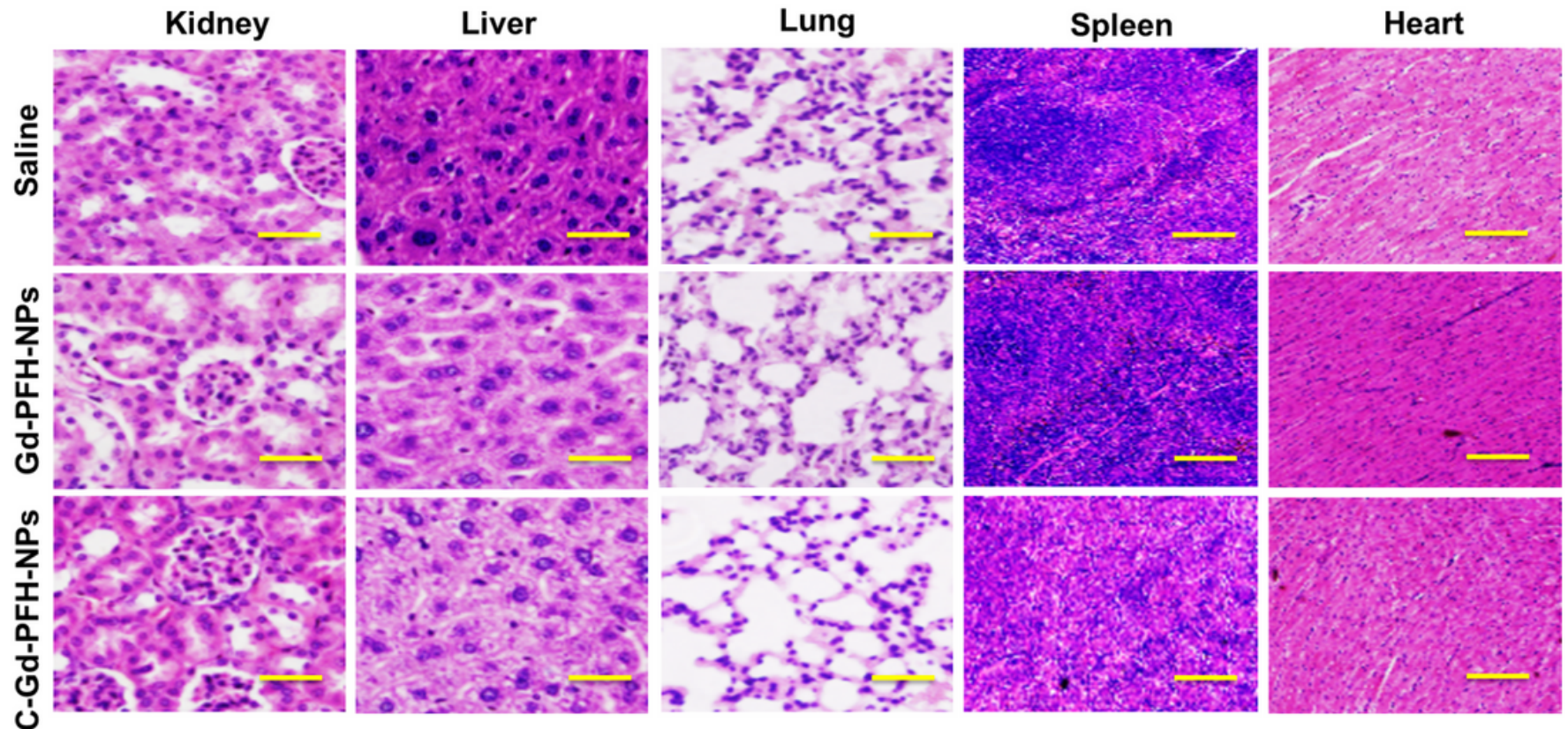

Figure 6 
H\&E staining of the major organs (kidney, liver, lung, spleen and heart) excised from different treatment mice groups. Scale bar: $100 \mu \mathrm{m}$.
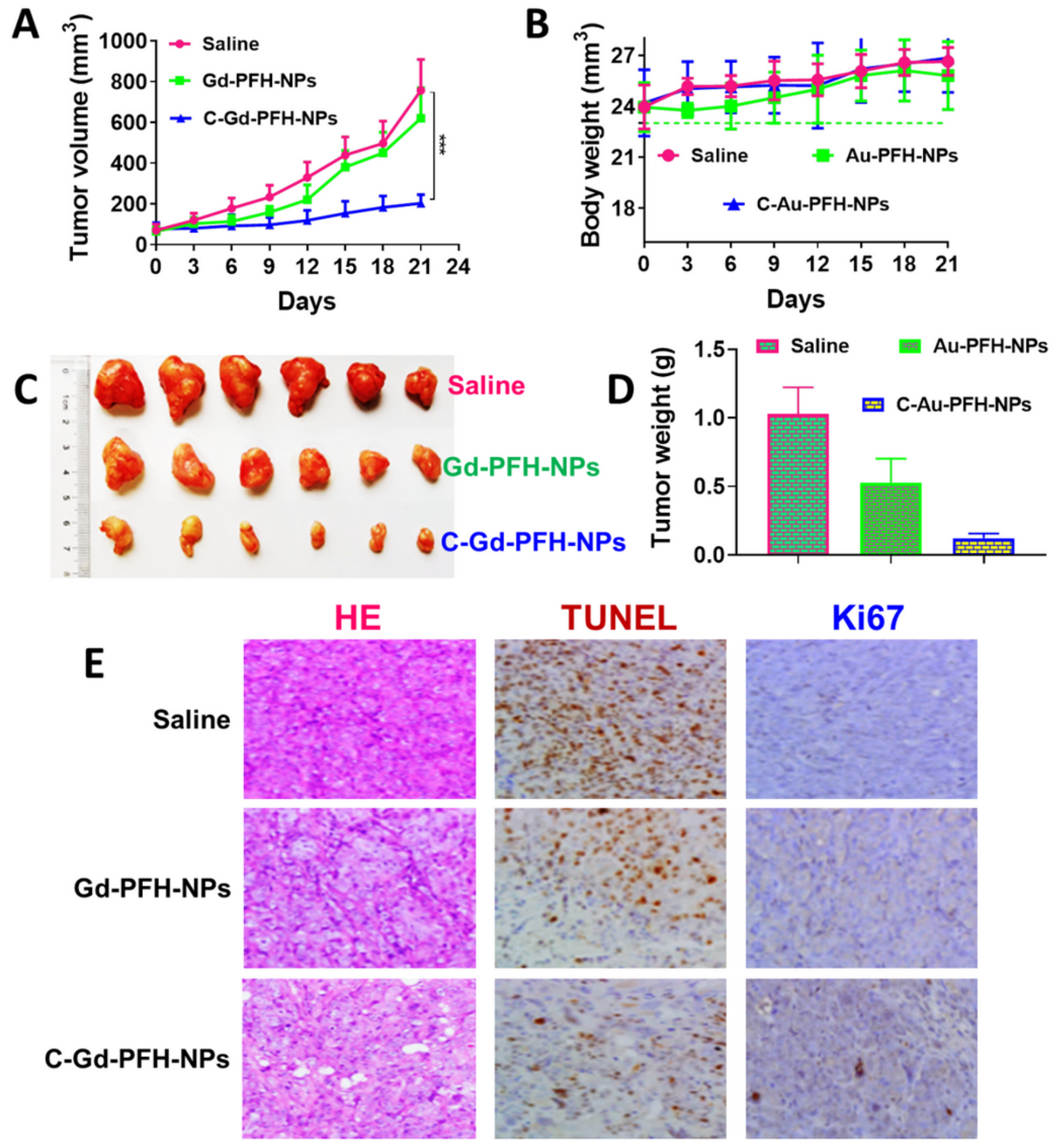

Figure 7

In vivo antitumor activity of Saline, Gd-PFH-NPs, and C-Gd-PFH-NPs compared to saline. C643 tumor xenograft-bearing $B A L B / c$ nude mice were administered with various drugs via intravenous injection at days 0, 3 and 6. A) Changes in tumor volumes. B) Body weights. C) Represent tumor photograph. D) 
Tumor weights. The data are presented as the means \pm SD $(n=7)$. E) Representative H\&E staining, Ki67, and TUNEL histopathological analysis of the tumors.
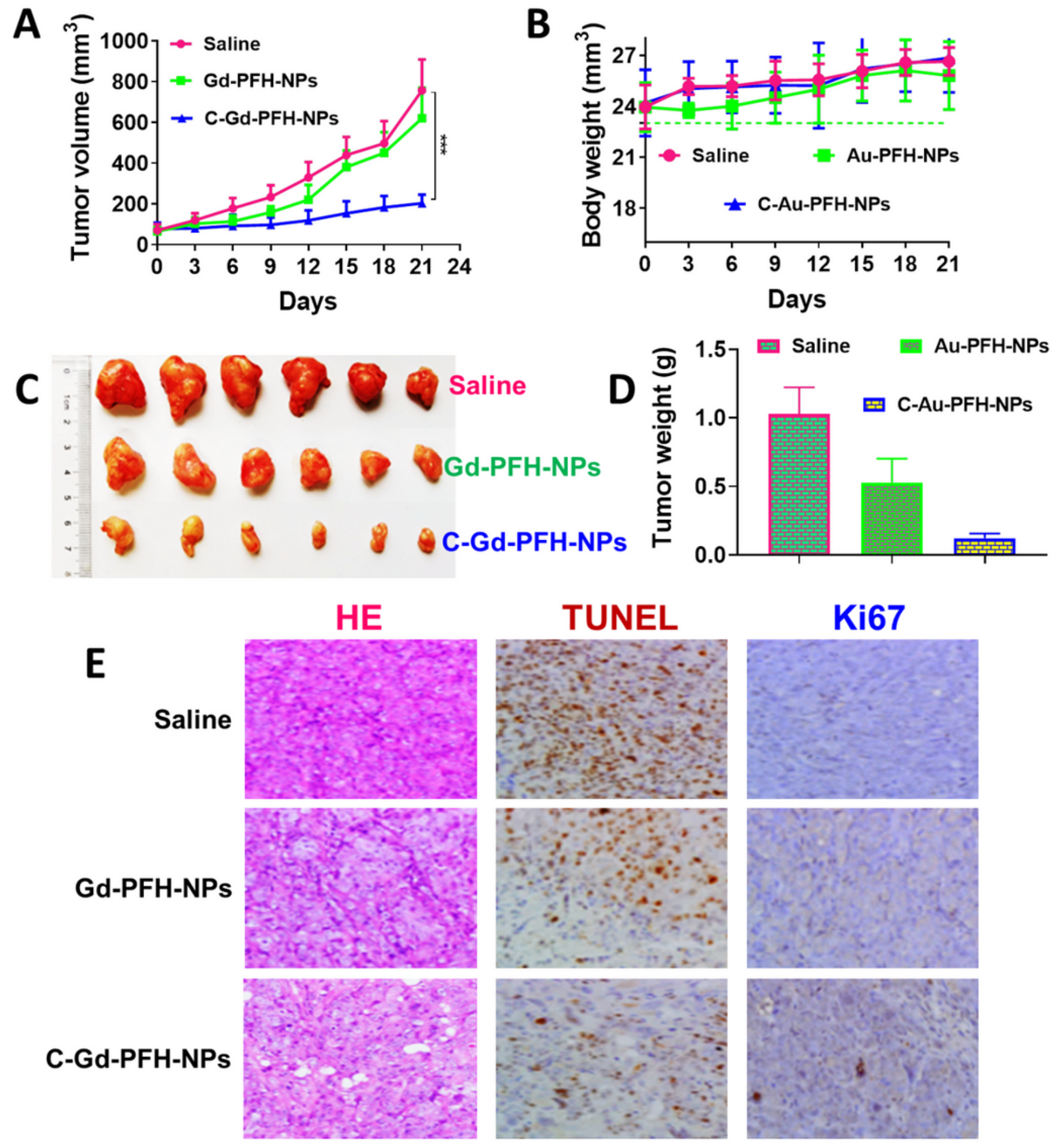

Figure 7

In vivo antitumor activity of Saline, Gd-PFH-NPs, and C-Gd-PFH-NPs compared to saline. C643 tumor xenograft-bearing BALB/c nude mice were administered with various drugs via intravenous injection at days 0, 3 and 6. A) Changes in tumor volumes. B) Body weights. C) Represent tumor photograph. D) 
Tumor weights. The data are presented as the means \pm SD $(n=7)$. E) Representative H\&E staining, Ki67, and TUNEL histopathological analysis of the tumors. 